\title{
Total Synthesis of Stachyodin A via One-Pot Suzuki Coupling for Quick Construction of Carbon Skeleton
}

\author{
Yuichiro Kawamoto, Toyoharu Kobayashi, and Hisanaka Ito* \\ School of Life Sciences, Tokyo University of Pharmacy and Life Sciences, 1432-1 \\ Horinouchi, Hachioji, Tokyo 192-0392, Japan \\ itohisa@toyaku.ac.jp
}

\section{Table of Contents}

$\begin{array}{ll}\text { Experimental procedure } & \mathrm{S} 2\end{array}$

${ }^{1} \mathrm{H}$ and ${ }^{13} \mathrm{C}$ NMR spectra of new compounds $\quad \mathrm{S} 14$

$\begin{array}{ll}\text { NOESY spectra of compound } \mathbf{S 2} / \mathbf{2 7 / S 4} & \text { S42 }\end{array}$

\section{General}

All reactions involving air- and moisture-sensitive reagents were carried out using standard syringe-septum cap techniques. Unless otherwise noted, all solvents and reagents were obtained from commercial suppliers and used without further purification. Routine monitoring of reactions was carried out Merck silica gel 60 F254 TLC plates. Column chromatography was performed on Kanto Chemical Silica Gel 60N (spherical, neutral 60-230 $\mu \mathrm{m}$ ) with the solvents indicated.

${ }^{1} \mathrm{H}$ and ${ }^{13} \mathrm{C}$ NMR spectra were measured with a JASCO ECZ $400 \mathrm{~S}$ (400 MHz) spectrometer. Chemical shifts were expressed in ppm using $\mathrm{CHCl}_{3}$ (7.26 ppm for ${ }^{1} \mathrm{H} \mathrm{NMR,} 77.0 \mathrm{ppm}$ for ${ }^{13} \mathrm{C}$ $\mathrm{NMR}$ ) in $\mathrm{CDCl}_{3}, \mathrm{C}_{6} \mathrm{H}_{6}$ (7.16 ppm for ${ }^{1} \mathrm{H} \mathrm{NMR}, 128.0 \mathrm{ppm}$ for ${ }^{13} \mathrm{C} \mathrm{NMR}$ ) in $\mathrm{C}_{6} \mathrm{D}_{6}$ and $\mathrm{CH}_{3} \mathrm{OH}$ (3.31 ppm for 1H NMR, $49.0 \mathrm{ppm}$ for $13 \mathrm{C} \mathrm{NMR}$ ) in $\mathrm{CD}_{3} \mathrm{OD}$ as internal standard. Infrared spectral measurements were carried out with a JASCO FT/IR-4700 and only noteworthy absorptions were listed. HRMS spectra measured on a Micromass LCT spectrometer. Oil bath was used in reactions that require heating and each temperature indicates oil bath temperature. 
3-bromo-4-(methoxymethoxy)cyclohex-2-en-1-one (15)<smiles>O=C1C=C(Br)C(O)CC1</smiles>

14 (racemic)

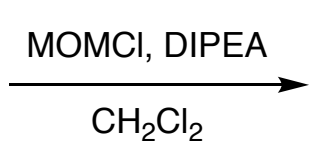

$87 \%$<smiles>COC1CCC(=O)C=C1Br</smiles>

15

To a solution of $\mathbf{1 4}(1.81 \mathrm{~g}, 9.48 \mathrm{mmol})$ in $\mathrm{CH}_{2} \mathrm{Cl}_{2}(20 \mathrm{~mL})$ was added DIPEA (15.7 ml, 94.8 mmol) and $\mathrm{MOMCl}(7.20 \mathrm{ml}, 94.8 \mathrm{mmol})$ at $0{ }^{\circ} \mathrm{C}$ under $\mathrm{Ar}$ atmosphere, and the mixture was stirred for $12 \mathrm{~h}$ at room temperature. The reaction mixture was quenched with $\mathrm{H}_{2} \mathrm{O}$, and extracted with $\mathrm{CHCl}_{3}$ (twice). The combined organic layers were washed with brine, dried over $\mathrm{MgSO}_{4}$, and concentrated in vacuo. The resulting residue was purified by silica gel column chromatography (hexane-AcOEt, 3:2) to afford 15 (1.93 g, 87\%) as colorless oil.

IR (neat) 2953, 1610, 1279, 1237, 1190, 1098, $927 \mathrm{~cm}^{-1} ;{ }^{1} \mathrm{H}$ NMR $\left(\mathrm{CDCl}_{3}, 400 \mathrm{MHz}\right) \delta 6.24$ (s, $1 \mathrm{H}), 4.82(\mathrm{~d}, 1 \mathrm{H}, J=7.2 \mathrm{~Hz}), 4.79$ (d, 1H, $J=7.2 \mathrm{~Hz}), 4.38(\mathrm{t}, 1 \mathrm{H}, J=4.8 \mathrm{~Hz}), 3.46$ (s, 3H), 2.61$2.71(\mathrm{~m}, 1 \mathrm{H}), 2.36-2.43(\mathrm{~m}, 1 \mathrm{H}), 2.16-2.29(\mathrm{~m}, 2 \mathrm{H}) ;{ }^{13} \mathrm{C} \mathrm{NMR}\left(\mathrm{CDCl}_{3}, 400 \mathrm{MHz}\right) \delta 195.6,148.8$, 133.9, 96.2, 74.3, 56.1, 33.0, 28.4; HRMS (ESI-TOF) $\mathrm{m} / \mathrm{z}$ : $[\mathrm{M}+\mathrm{Na}]^{+} \mathrm{Calcd}$ for $\mathrm{C}_{8} \mathrm{H}_{11} \mathrm{BrO}_{3} \mathrm{Na}$ 256.9789; Found 256.9785.

$(((1 R, 4 S)$-3-bromo-4-(methoxymethoxy)cyclohex-2-en-1-yl)oxy)(tertbutyl)dimethylsilane (16)<smiles>COC1CCC(=O)C=C1Br</smiles>

15<smiles>CO[C@H]1CC[C@@H](C)C=C1Br</smiles>

S1

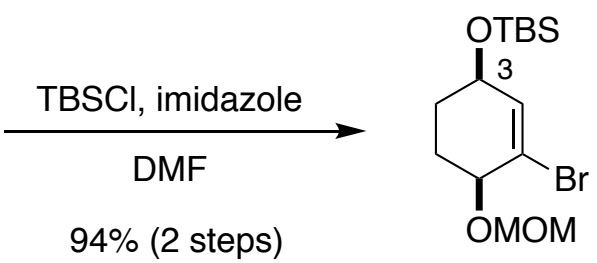

16

(5:1 mixture at C3)

To a solution of $15(1.93 \mathrm{~g}, 8.21 \mathrm{mmol})$ in THF $(20 \mathrm{~mL})$ was added $\mathrm{LiAlH}(\mathrm{O} t-\mathrm{Bu})_{3}(1.0 \mathrm{M}$ in THF, $9.85 \mathrm{ml}, 9.85 \mathrm{mmol}$ ) under Ar atmosphere, and the mixture was stirred for $30 \mathrm{~min}$ at room temperature. The reaction mixture was quenched with sat. $\mathrm{NH}_{4} \mathrm{Cl}$ aq., and extracted with $\mathrm{AcOEt}$ (twice). The combined organic layers were washed with brine, dried over $\mathrm{MgSO}_{4}$, and concentrated in vacuo to give crude $\mathbf{S 1}$ as 5:1 diastereomer mixture at $\mathrm{C} 3$. To a solution of crude 
S1 in DMF (10 ml) was added imidazole $(3.07 \mathrm{~g}, 45.2 \mathrm{mmol})$ and TBSCl $(6.19 \mathrm{~g}, 41.1 \mathrm{mmol})$ at $0{ }^{\circ} \mathrm{C}$ under $\mathrm{Ar}$ atmosphere, and the mixture was stirred for $12 \mathrm{~h}$ at room temperature. The reaction mixture was quenched with $\mathrm{H}_{2} \mathrm{O}$, and extracted with AcOEt (twice). The combined organic layers were washed with brine, dried over $\mathrm{MgSO}_{4}$, and concentrated in vacuo. The resulting residue was purified by silica gel column chromatography (hexane-AcOEt, 92:8) to afford $\mathbf{1 6}$ ( $2.71 \mathrm{~g}, 94 \%$ in 2 steps) as 5:1 diastereomer mixture at $\mathrm{C} 3$ as colorless oil.

IR (neat) 2952, 2857, 1471, 1366, 1254, 1150, 1097, 1035, 983, 944, 920, 891, 867, 837, $776 \mathrm{~cm}^{-}$ ${ }^{1}$; ${ }^{1} \mathrm{H}$ NMR for only major isomer $\left(\mathrm{CDCl}_{3}, 400 \mathrm{MHz}\right) \delta 6.16(\mathrm{~d}, 1 \mathrm{H}, J=1.2 \mathrm{~Hz}), 4.74(\mathrm{~s}, 2 \mathrm{H})$, 4.12-4.19 (m, 1H), 4.05 (t, 1H, $J=3.2 \mathrm{~Hz}), 3.45$ (s, 3H), 1.96-2.07 (m, 1H), 1.70-1.80 (m, 3H), $0.87(9 \mathrm{H}, \mathrm{s}), 0.06(3 \mathrm{H}, \mathrm{s}), 0.05(3 \mathrm{H}, \mathrm{s}) ;{ }^{13} \mathrm{C} \mathrm{NMR}$ for only major isomer $\left(\mathrm{CDCl}_{3}, 400 \mathrm{MHz}\right) \delta$ 137.9, 124.0, 96.0, 74.4, 69.0, 55.9, 27.2, 27.1, 25.8, 25.7, 18.0, -4.6, -4.7; HRMS (ESI-TOF) m/z: [M+Na] $]^{+}$Calcd for $\mathrm{C}_{14} \mathrm{H}_{27} \mathrm{BrO}_{3} \mathrm{SiNa} 373.0811$; Found 373.0811 .

(1R,4S)-3-bromo-4-(methoxymethoxy)cyclohex-2-en-1-yl 4-bromobenzoate (S2)<smiles>COC1CCC(O)C=C1Br</smiles>

S1

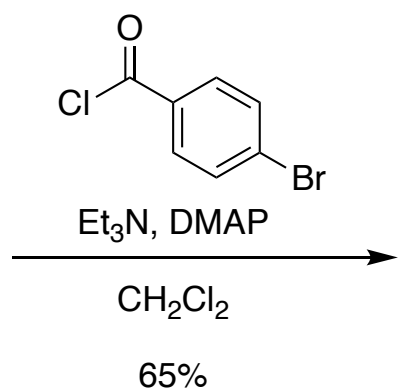

$65 \%$<smiles>COC1CCC(OC(=O)c2ccc(Br)cc2)C=C1Br</smiles>

S2

To a solution of $\mathbf{S 1}(52.0 \mathrm{mg}, 0.219 \mathrm{mmol})$ in $\mathrm{CH}_{2} \mathrm{Cl}_{2}(3 \mathrm{~mL})$ was added $\mathrm{Et}_{3} \mathrm{~N}(61.0 \mathrm{ul}, 0.438$ mmol), $p$-bromobenzoylchloride $(96.0 \mathrm{mg}, 0.438 \mathrm{mmol})$ and DMAP $(5.40 \mathrm{mg}, 0.0438 \mathrm{mmol})$ under Ar atmosphere, and the mixture was stirred for $5 \mathrm{~h}$ at room temperature. The reaction mixture was quenched with $\mathrm{H}_{2} \mathrm{O}$ and extracted with $\mathrm{CHCl}_{3}$ (twice). The combined organic layers were washed with brine, dried over $\mathrm{MgSO}_{4}$, and concentrated in vacuo. The resulting residue was purified by silica gel column chromatography (hexane-AcOEt, 3:1) to afford $\mathbf{S 2}(60 \mathrm{mg}, 65 \%$ ) as diastereomer mixture at $\mathrm{C} 3$ as colorless oil.

IR (neat) 2951, 1718, 1589, 1266, 1150, 1100, 1032, 1011, $756 \mathrm{~cm}^{-1} ;{ }^{1} \mathrm{H}$ NMR for only major isomer $\left(\mathrm{CDCl}_{3}, 400 \mathrm{MHz}\right) \delta 7.90(\mathrm{~d}, 2 \mathrm{H}, J=8.4 \mathrm{~Hz}), 7.58(\mathrm{~d}, 2 \mathrm{H}, J=8.4 \mathrm{~Hz}), 6.36(\mathrm{~d}, 1 \mathrm{H}, J=$ 2.8 Hz), 5.43-5.49 (m, 1H), 4.79 (s, 2H), 4.18 (t, 1H, J=4.0 Hz), 3.49 (s, 3H), 1.94-2.22 (m, 4H); ${ }^{13} \mathrm{C}$ NMR for only major isomer $\left(\mathrm{CDCl}_{3}, 400 \mathrm{MHz}\right) \delta 132.0,131.7,131.2,128.8,128.3,128.2$, 
96.2, 76.7, 74.2, 70.4, 56.0, 27.1, 23.4; HRMS (ESI-TOF) $\mathrm{m} / \mathrm{z}:[\mathrm{M}+\mathrm{Na}]^{+}$Calcd for $\mathrm{C}_{15} \mathrm{H}_{16} \mathrm{Br}_{2} \mathrm{O}_{4} \mathrm{Na} 440.9313$; Found 440.9307.

tert-butyl(((1R,4S)-4-(methoxymethoxy)-3-(4,4,5,5-tetramethyl-1,3,2-dioxaborolan-2yl)cyclohex-2-en-1-yl)oxy)dimethylsilane (17)<smiles>CO[C@H]1CC[C@@H](O[SbH3])C=C1Br</smiles>

16

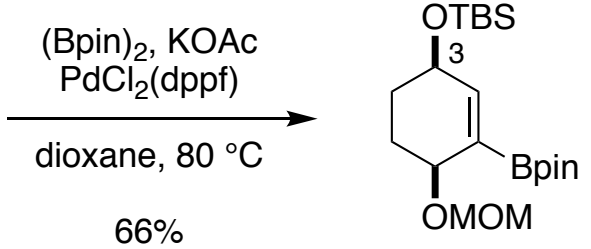

17

(5:1 mixture at C3)

To a solution of $16(112 \mathrm{mg}, 0.320 \mathrm{mmol})$ in dioxane $(3 \mathrm{ml})$ was added (Bpin) $)_{2}(240 \mathrm{mg}, 0.640$ mmol), $\mathrm{PdCl}_{2}$ (dppf) $(69.0 \mathrm{mg}, 0.0640 \mathrm{mmol})$ and $\mathrm{KOAc}(139 \mathrm{mg}, 0.960 \mathrm{mmol})$ under $\mathrm{Ar}$ atmosphere, and the mixture was stirred for $2 \mathrm{~h}$ at $70^{\circ} \mathrm{C}$. The reaction mixture was quenched with brine, and extracted with $\mathrm{CHCl}_{3}$ (twice). The combined organic layers were washed with brine, dried over $\mathrm{MgSO}_{4}$, and concentrated in vacuo. The resulting residue was purified by silica gel column chromatography (hexane-AcOEt, 92:8) to afford $1784.0 \mathrm{mg}, 66 \%$ ) as 5:1 diastereomer mixture at $\mathrm{C} 3$ as pale yellow oil.

IR (neat) 2931, 1365, 1317, 1254, 1148, 1099, 1036, 880, 838, $775 \mathrm{~cm}^{-1}$; ${ }^{1} \mathrm{H}$ NMR for only major isomer $\left(\mathrm{CDCl}_{3}, 400 \mathrm{MHz}\right) \delta 6.47(\mathrm{~s}, 1 \mathrm{H}), 4.80(\mathrm{~d}, 1 \mathrm{H}, J=6.8 \mathrm{~Hz}), 4.69(\mathrm{~d}, 1 \mathrm{H}, J=6.8 \mathrm{~Hz}), 4.09-$ $4.15(\mathrm{~m}, 2 \mathrm{H}), 3.40(\mathrm{~s}, 3 \mathrm{H}), 1.91-1.98(\mathrm{~m}, 1 \mathrm{H}), 1.71-1.83(\mathrm{~m}, 2 \mathrm{H}), 1.52-1.60(\mathrm{~m}, 1 \mathrm{H}), 1.25(\mathrm{~s}$, $12 \mathrm{H}), 0.88(\mathrm{~s}, 9 \mathrm{H}), 0.07$ (s, 3H), $0.06(\mathrm{~s}, 3 \mathrm{H}) ;{ }^{13} \mathrm{C} \mathrm{NMR}$ for only major isomer $\left(\mathrm{CDCl}_{3}, 400 \mathrm{MHz}\right)$ $\delta 148.9$ (overlapping of 2 peaks), 96.1, 83.3, 69.9, 68.3, 55.4, 27.4, 26.9, 25.8, 24.8, 24.7, 18.1, 4.5, -4.6; HRMS (ESI-TOF) m/z: [M+Na] ${ }^{+}$Calcd for $\mathrm{C}_{20} \mathrm{H}_{39} \mathrm{BO}_{5} \mathrm{SiNa} 421.2558$; Found 421.2557.

ethyl (E)-3-((3R,6S)-3-((tert-butyldimethylsilyl)oxy)-6-(methoxymethoxy)cyclohex-1en-1-yl)-2-(4-methoxy-2-(methoxymethoxy)phenyl)acrylate (21) 


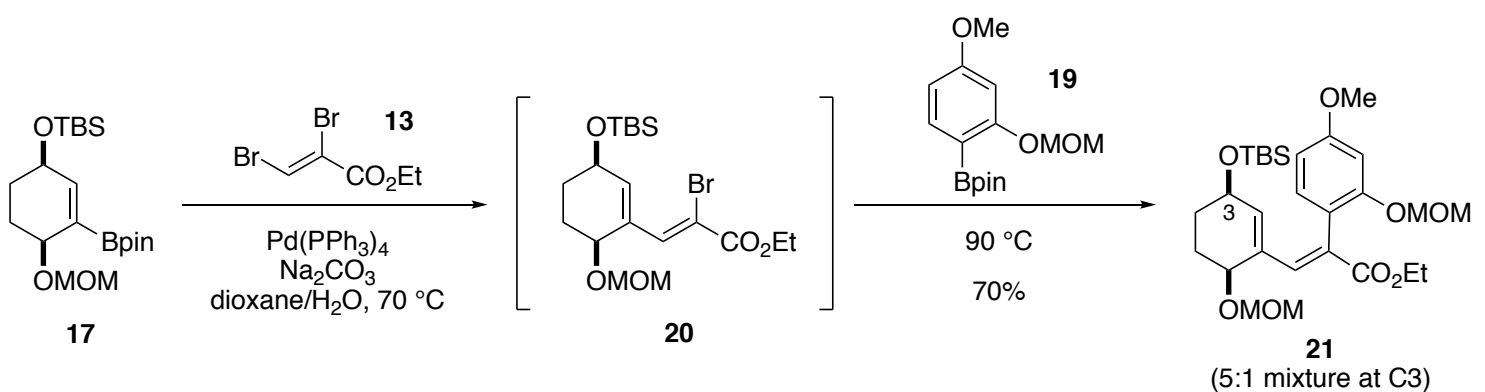

To a solution of $17(19.0 \mathrm{mg}, 0.0477 \mathrm{mmol})$ and $13(25.0 \mathrm{mg}, 0.0969 \mathrm{mmol})$ in dioxane $(1 \mathrm{ml})$ was added $\mathrm{Pd}\left(\mathrm{PPh}_{3}\right)_{4}(11.0 \mathrm{mg}, 0.00952 \mathrm{mmol})$ and $\mathrm{Na}_{2} \mathrm{CO}_{3}(10.0 \mathrm{mg}, 0.0943 \mathrm{mmol})$ in $\mathrm{H}_{2} \mathrm{O}(0.3$ $\mathrm{ml}$ ) under Ar atmosphere, and the mixture was stirred for $4 \mathrm{~h}$ at $70{ }^{\circ} \mathrm{C}$. After cooling, to the reaction mixture was added $19(28.0 \mathrm{mg}, 0.0952 \mathrm{mmol}), \mathrm{Pd}\left(\mathrm{PPh}_{3}\right)_{4}(11.0 \mathrm{mg}, 0.00952 \mathrm{mmol})$ and $\mathrm{Na}_{2} \mathrm{CO}_{3}(15.0 \mathrm{mg}, 0.142 \mathrm{mmol})$ in $\mathrm{H}_{2} \mathrm{O}(0.3 \mathrm{ml})$, and the mixture was stirred for $4 \mathrm{~h}$ at $90^{\circ} \mathrm{C}$. The reaction mixture was quenched with brine, and extracted with AcOEt (twice). The combined organic layers were washed with brine, dried over $\mathrm{MgSO}_{4}$, and concentrated in vacuo. The resulting residue was purified by silica gel column chromatography (hexane-AcOEt, 4:1) to afford $21(18.0 \mathrm{mg}, 70 \%)$ as 5:1 diastereomer mixture at $\mathrm{C} 3$ as pale yellow oil.

IR (neat) 2952, 1711, 1606, 1508, 1239, 1153, 1079, 1031, $837 \mathrm{~cm}^{-1} ;{ }^{1} \mathrm{H}$ NMR for only major isomer $\left(\mathrm{CDCl}_{3}, 400 \mathrm{MHz}\right) \delta 7.26\left(\mathrm{~s}, 1 \mathrm{H}\right.$, overlapping with peak of $\left.\mathrm{CHCl}_{3}\right), 7.01(\mathrm{~d}, 1 \mathrm{H}, J=8.8$ $\mathrm{Hz}), 6.74$ (d, 1H, $J=2.4 \mathrm{~Hz}), 6.53$ (dd, 1H, $J=2.4 \mathrm{~Hz}, 8.8 \mathrm{~Hz}), 5.60$ (d, 1H, $J=2.8 \mathrm{~Hz}), 5.09$ (d, $1 \mathrm{H}, J=6.8 \mathrm{~Hz}), 5.07(\mathrm{~d}, 1 \mathrm{H}, J=6.8 \mathrm{~Hz}), 4.66(\mathrm{~d}, 1 \mathrm{H}, J=6.8 \mathrm{~Hz}), 4.46(\mathrm{~d}, 1 \mathrm{H}, J=6.8 \mathrm{~Hz}), 4.18$ (qd, 2H, $J=1.2 \mathrm{~Hz}, 6.8 \mathrm{~Hz}), 3.99$ (t, 1H, $J=6.0 \mathrm{~Hz}), 3.93$ (t, 1H, $J=3.2 \mathrm{~Hz}), 3.78(\mathrm{~s}, 3 \mathrm{H}), 3.41$ (s, 6H), 1.91-1.98 (m, 1H), 1.45-1.68 (m, 3H), 1.23 (t, 3H, $J=6.8 \mathrm{~Hz}), 0.79$ (s, 9H), -0.07 (s, 3H), $-0.11(\mathrm{~s}, 3 \mathrm{H}) ;{ }^{13} \mathrm{C} \mathrm{NMR}$ for only major isomer $\left(400 \mathrm{MHz}, \mathrm{CDCl}_{3}\right) \delta 167.9,160.5,156.2,139.6$, 139.3, 133.6, 131.1, 129.9, 118.4, 106.7, 101.7, 96.0, 94.8, 71.7, 67.7, 60.7, 55.9, 55.6, 55.2, 26.9, 26.1, 25.7, 18.0, 14.3, -4.9, -5.0; HRMS (ESI-TOF) $\mathrm{m} / \mathrm{z}$ : $[\mathrm{M}+\mathrm{Na}]^{+}$Calcd for $\mathrm{C}_{28} \mathrm{H}_{44} \mathrm{O}_{8} \mathrm{SiNa}$ 559.2703 ; Found 559.2708.

(E)-3-((3R,6S)-3-((tert-butyldimethylsilyl)oxy)-6-(methoxymethoxy)cyclohex-1-en-1yl)-2-(4-methoxy-2-(methoxymethoxy)phenyl)prop-2-en-1-ol (22) 
<smiles>CCOC(=O)C(=CC1=CC([OH2+])CCC1OC)c1ccc(OC)cc1OC</smiles>

21

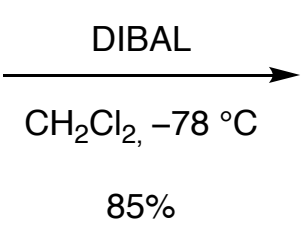

$85 \%$<smiles>COc1ccc(OC)c(/C(=C/C2=C[C@@H](OC)CCC2OC)CO)c1</smiles>

22

(5:1 mixture at C3)

To a solution of $21(50.0 \mathrm{mg}, 0.0932 \mathrm{mmol})$ in $\mathrm{CH}_{2} \mathrm{Cl}_{2}(2 \mathrm{ml})$ was added DIBAL (1.01 $\mathrm{M}$ in toluene, $92.0 \mathrm{ul}, 0.280 \mathrm{mmol}$ ) at $-78^{\circ} \mathrm{C}$ under $\mathrm{Ar}$ atmosphere, and the mixture was stirred for $2 \mathrm{~h}$ at the same temperature. The reaction mixture was quenched with sat. $\mathrm{NH}_{4} \mathrm{Cl}$ aq. at $-78{ }^{\circ} \mathrm{C}$, allowed to warm to room temperature, and extracted with $\mathrm{CHCl}_{3}$ (twice). The combined organic layers were washed with brine, dried over $\mathrm{MgSO}_{4}$, and concentrated in vacuo. The resulting residue was purified by silica gel column chromatography (hexane-AcOEt, 1:1) to afford $22(39.0$ $\mathrm{mg}, 85 \%)$ as 5:1 diastereomer mixture at $\mathrm{C} 3$ as pale yellow oil.

IR (neat) 3453, 2927, 1607, 1504, 1464, 1256, 1217, 1153, 1075, 1031, 918, 836, $776 \mathrm{~cm}^{-1} ;{ }^{1} \mathrm{H}$ NMR for only major isomer $\left(\mathrm{C}_{6} \mathrm{D}_{6}, 400 \mathrm{MHz}\right) \delta 7.09(\mathrm{~d}, 1 \mathrm{H}, J=8.4 \mathrm{~Hz}), 6.93(\mathrm{~d}, 1 \mathrm{H}, J=2.4 \mathrm{~Hz})$, $6.46(\mathrm{dd}, 1 \mathrm{H}, J=2.4 \mathrm{~Hz}, 8.4 \mathrm{~Hz}), 6.42(\mathrm{~s}, 1 \mathrm{H}), 5.63(\mathrm{~d}, 1 \mathrm{H}, J=2.0 \mathrm{~Hz}), 4.88(\mathrm{~s}, 2 \mathrm{H}), 4.63(\mathrm{~d}, 1 \mathrm{H}$, $J=7.2 \mathrm{~Hz}), 4.54$ (d, 1H, $J=7.2 \mathrm{~Hz}), 4.36$ (d, 1H, $J=5.6 \mathrm{~Hz}), 3.99-4.05$ (m, 1H), 3.96 (t, 1H, $J$ $=3.2 \mathrm{~Hz}), 3.36(\mathrm{~s}, 3 \mathrm{H}), 3.29(\mathrm{~s}, 3 \mathrm{H}), 3.18(\mathrm{~s}, 3 \mathrm{H}), 1.84-1.95(\mathrm{~m}, 2 \mathrm{H}), 1.54-1.63(\mathrm{~m}, 1 \mathrm{H}), 1.33-$ $1.42(\mathrm{~m}, 1 \mathrm{H}), 0.94(\mathrm{~s}, 9 \mathrm{H}),-0.01(\mathrm{~s}, 3 \mathrm{H}),-0.03(\mathrm{~s}, 3 \mathrm{H}) ;{ }^{13} \mathrm{C}$ NMR for only major isomer $\left(\mathrm{C}_{6} \mathrm{D}_{6}\right.$, $400 \mathrm{MHz}) \delta 160.8,156.1,139.9,135.0,133.9,131.4,126.7,121.6,107.3,102.4,96.1,94.9,72.8$, 68.4, 67.9, 55.8, 55.4, 54.8, 27.8, 26.7, 26.0, 18.3, -4.7; HRMS (ESI-TOF) m/z: [M+Na] ${ }^{+} \mathrm{Calcd}$ for $\mathrm{C}_{26} \mathrm{H}_{42} \mathrm{O}_{7} \mathrm{SiNa}$ 517.2597; Found 517.2593.

((2R,3R)-3-((3R,6S)-3-((tert-butyldimethylsilyl)oxy)-6-(methoxymethoxy)cyclohex-1en-1-yl)-2-(4-methoxy-2-(methoxymethoxy)phenyl)oxiran-2-yl)methanol (23) 
<smiles>COc1cc(OC)c(/C(=C/C2=CC([O+])CCC2OC)CO)cc1OC</smiles>

22

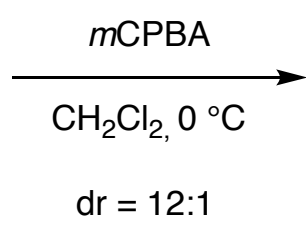

$r=12: 1$<smiles>COc1ccc([C@]2(CO)O[C@@H]2C2=C[C@@H](O)CCC2OC)c(OC)c1</smiles>

23

$90 \%$ (minor isomer at C6a-C11a was removed by column separation)

To a solution of $22(28.0 \mathrm{mg}, 0.0566 \mathrm{mmol})$ in $\mathrm{CH}_{2} \mathrm{Cl}_{2}(1.2 \mathrm{ml})$ was added $m \mathrm{CPBA}$ (14.7 $\mathrm{mg}$, $0.0852 \mathrm{mmol}$ ) at $0{ }^{\circ} \mathrm{C}$ under $\mathrm{Ar}$ atmosphere, and the mixture was stirred for $1 \mathrm{~h}$ at the same temperature. The reaction mixture was quenched with sat. $\mathrm{Na}_{2} \mathrm{~S}_{2} \mathrm{O}_{3}$ aq., allowed to warm to room temperature, and extracted with $\mathrm{CHCl}_{3}$ (twice). The combined organic layers were washed with brine, dried over $\mathrm{MgSO}_{4}$, and concentrated in vacuo. The resulting residue was purified by silica gel column chromatography (hexane-AcOEt, 1:1) to afford $\mathbf{2 3}(26.0 \mathrm{mg}, 90 \%)$ as 5:1 diastereomer mixture at $\mathrm{C} 3$ (minor isomer at $\mathrm{C} 6 \mathrm{a}-\mathrm{C} 12 \mathrm{a}$ was removed by column separation), and as colorless oil.

IR (neat) 3435, 2952, 1614, 1509, 1470, 1256, 1218, 1154, 1075, 1031, 921, 836, $775 \mathrm{~cm}^{-1} ;{ }^{1} \mathrm{H}$ NMR -preparative TLC separated 5:1 mixture $\mathbf{2 3}$ to isolate major isomer $\mathbf{2 3}$ ' for ${ }^{1} \mathrm{H}$ data- $\left(\mathrm{C}_{6} \mathrm{D}_{6}\right.$, $400 \mathrm{MHz}) \delta 7.51(\mathrm{~d}, 1 \mathrm{H}, J=8.4 \mathrm{~Hz}), 6.95(\mathrm{~d}, 1 \mathrm{H}, J=2.4 \mathrm{~Hz}), 6.47(\mathrm{dd}, 1 \mathrm{H}, J=2.4 \mathrm{~Hz}, 8.4 \mathrm{~Hz})$, $5.54(\mathrm{~d}, 1 \mathrm{H}, J=2.4 \mathrm{~Hz}), 4.97(\mathrm{~d}, 1 \mathrm{H}, J=2.8 \mathrm{~Hz}), 4.91(\mathrm{~d}, 1 \mathrm{H}, J=2.8 \mathrm{~Hz}), 4.63(\mathrm{~d}, 1 \mathrm{H}, J=2.8$ Hz), 4.59 (d, 1H, $J=2.8 \mathrm{~Hz}), 3.87-4.11(\mathrm{~m}, 5 \mathrm{H}), 3.35$ (s, 3H), $3.34(\mathrm{~s}, 3 \mathrm{H}), 3.21(\mathrm{~s}, 3 \mathrm{H}), 1.61-$ $1.79(\mathrm{~m}, 3 \mathrm{H}), 1.43-1.52(\mathrm{~m}, 1 \mathrm{H}), 0.91(\mathrm{~s}, 9 \mathrm{H}),-0.04(\mathrm{~s}, 3 \mathrm{H}),-0.07(\mathrm{~s}, 3 \mathrm{H}) ;{ }^{13} \mathrm{C}$ NMR for only major isomer $\left(\mathrm{C}_{6} \mathrm{D}_{6}, 400 \mathrm{MHz}\right) \delta 161.2,157.4,133.9,131.9,131.1,116.9,106.4,102.0,95.7$, 95.1, 71.6, 67.6, 66.8, 64.3, 59.4, 55.6, 55.3, 54.7, 28.6, 26.1, 26.0, 18.2, -4.7; HRMS (ESI-TOF) $\mathrm{m} / \mathrm{z}:[\mathrm{M}+\mathrm{Na}]^{+}$Calcd for $\mathrm{C}_{26} \mathrm{H}_{42} \mathrm{O}_{8} \mathrm{SiNa}$ 533.2547; Found 533.2550.

(1S,2R)-1-((3R,6S)-3-((tert-butyldimethylsilyl)oxy)-6-(methoxymethoxy)cyclohex-1en-1-yl)-2-(4-methoxy-2-(methoxymethoxy)phenyl)propane-1,3-diol (24) 
<smiles>COc1ccc(C(=O)CC2=CC([O+])CCC2OC)c(OC)c1</smiles>

23

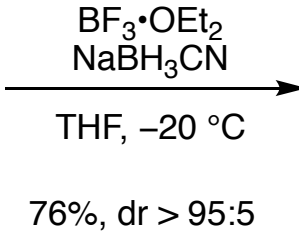

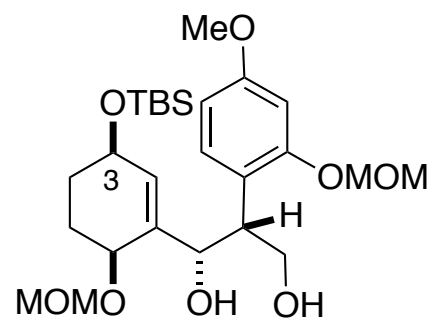

24

(5:1 mixture at C3)

To a solution of $23(41.0 \mathrm{mg}, 0.0803 \mathrm{mmol})$ in THF $(2 \mathrm{ml})$ was added $\mathrm{NaBH}_{3} \mathrm{CN}(50.0 \mathrm{mg}, 0.796$ mmol) and $\mathrm{BF}_{3} \cdot \mathrm{OEt}_{2}(40.0 \mathrm{ul}, 0.318 \mathrm{mmol})$ at $-20{ }^{\circ} \mathrm{C}$ under $\mathrm{Ar}$ atmosphere, and the mixture was stirred for $1 \mathrm{~h}$ at the same temperature. The reaction mixture was quenched with sat. $\mathrm{NaHCO}_{3}$ aq., allowed to warm to room temperature, and extracted with $\mathrm{CHCl}_{3}$ (twice). The combined organic layers were washed with brine, dried over $\mathrm{MgSO}_{4}$, and concentrated in vacuo. The resulting residue was purified by silica gel column chromatography (hexane-AcOEt, 3:7) to afford 24 (31.0 $\mathrm{mg}, 76 \%$ ) as 5:1 diastereomer mixture at $\mathrm{C} 3$ as colorless oil. The relative stereochemistry of diol 24 was confirmed by NOESY experiment of the corresponding acetonide S4 (see page S44) IR (neat) 3456, 2952, 1611, 1506, 1470, 1255, 1216, 1153, 920, 836, $776 \mathrm{~cm}^{-1} ;{ }^{1} \mathrm{H}$ NMR $\left(\mathrm{C}_{6} \mathrm{D}_{6}\right.$, $400 \mathrm{MHz}) \delta 7.54(\mathrm{~d}, 1 \mathrm{H}, J=8.8 \mathrm{~Hz}), 6.94(\mathrm{~d}, 1 \mathrm{H}, J=2.4 \mathrm{~Hz}), 6.54(\mathrm{dd}, 1 \mathrm{H}, J=2.4 \mathrm{~Hz}, 8.8 \mathrm{~Hz})$, $5.89(\mathrm{~d}, 1 \mathrm{H}, J=2.4 \mathrm{~Hz}), 4.91(\mathrm{~d}, 1 \mathrm{H}, J=6.8 \mathrm{~Hz}), 4.86(\mathrm{~d}, 1 \mathrm{H}, J=6.8 \mathrm{~Hz}), 4.78-4.82(\mathrm{~m}, 1 \mathrm{H})$, 4.44 (d, 1H, $J=6.4 \mathrm{~Hz}), 4.40$ (d, 1H, $J=6.4 \mathrm{~Hz}), 4.13-4.19$ (m, 1H), 4.05-4.11 (m, 1H), 3.954.03 (m, 1H), 3.81-3.95 (m, 2H), 3.36 (s, 3H), 3.17 (s, 3H), 3.13 (s, 3H), 1.84-1.95 (m, 2H), 1.56$1.63(\mathrm{~m}, 1 \mathrm{H}), 1.32-1.40(\mathrm{~m}, 1 \mathrm{H}), 0.98(\mathrm{~s}, 9 \mathrm{H}), 0.07(\mathrm{~s}, 3 \mathrm{H}), 0.06(\mathrm{~s}, 3 \mathrm{H}) ;{ }^{13} \mathrm{C}$ NMR for only major isomer $\left(400 \mathrm{MHz}, \mathrm{CDCl}_{3}\right) \delta 159.5,156.6,138.1,134.5,129.7,120.4,106.6,101.7,96.0,94.8$, 75.3, 71.8, 67.3, 64.3, 56.1, 55.3, 45.2, 27.6, 26.0, 25.8, 18.1, -4.5, -4.7; HRMS (ESI-TOF) m/z: $[\mathrm{M}+\mathrm{Na}]^{+}$Calcd for $\mathrm{C}_{26} \mathrm{H}_{44} \mathrm{O}_{8} \mathrm{SiNa}$ 535.2703; Found 535.2706.

$(1 S, 2 R)-1-((3 R, 6 S)-3-h y d r o x y-6-(m e t h o x y m e t h o x y) c y c l o h e x-1-e n-1-y l)-2-(4-m e t h o x y-$ 2-(methoxymethoxy)phenyl)propane-1,3-diol (25) 
<smiles>COc1ccc([C@H](CO)[C@@H](O)C2=CC([O+]C(C)(C)C)CCC2OC)c(OC)c1</smiles>

24

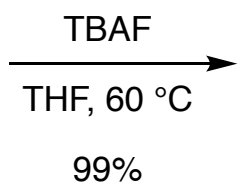

MOMO<smiles>COc1ccc([C@H](CO)[C@H](O)C2=C[C@H](O)CCC2OC)c(OC)c1</smiles>

25

(5:1 mixture at C3)

To a solution of $24(34.0 \mathrm{mg}, 0.0663 \mathrm{mmol})$ in THF $(2 \mathrm{ml})$ was added TBAF (1 M in THF, 663 $\mathrm{ul}, 0.663 \mathrm{mmol}$ ) at room temperature under Ar atmosphere, and the mixture was stirred for $1 \mathrm{~h}$ at $60{ }^{\circ} \mathrm{C}$. After cooling, the reaction mixture was concentrated in vacuo. The resulting residue was purified by silica gel column chromatography $\left(\mathrm{CHCl}_{3}-\mathrm{MeOH}, 93: 7\right)$ to afford $\mathbf{2 5}(26.0 \mathrm{mg}, 99 \%)$ as 5:1 diastereomer mixture at $\mathrm{C} 3$ as colorless oil.

IR (neat) 3409, 2938, 1610, 1585, 1507, 1443, 1283, 1216, 1152, 1072, 920, 841, $752 \mathrm{~cm}^{-1} ;{ }^{1} \mathrm{H}$ $\operatorname{NMR}\left(\mathrm{CDCl}_{3}, 400 \mathrm{MHz}\right) \delta 7.28(\mathrm{~d}, 1 \mathrm{H}, J=2.0 \mathrm{~Hz}), 6.71(\mathrm{~d}, 1 \mathrm{H}, J=2.0 \mathrm{~Hz}), 6.57(\mathrm{dd}, 1 \mathrm{H}, J=$ $2.0 \mathrm{~Hz}, 8.8 \mathrm{~Hz}), 5.94$ (d, 1H, $J=2.4 \mathrm{~Hz}), 5.19(\mathrm{~d}, 1 \mathrm{H}, J=6.8 \mathrm{~Hz}), 5.16(\mathrm{~d}, 1 \mathrm{H}, J=6.8 \mathrm{~Hz}), 4.73$ $(\mathrm{d}, 1 \mathrm{H}, J=6.8 \mathrm{~Hz}), 4.67$ (d, 1H, $J=6.8 \mathrm{~Hz}), 4.52(\mathrm{~d}, 1 \mathrm{H}, J=8.4 \mathrm{~Hz}), 4.10-4.30$ (m, 3H), 3.71$3.83(\mathrm{~m}, 4 \mathrm{H}), 3.54-3.62(\mathrm{~m}, 1 \mathrm{H}), 3.47(\mathrm{~s}, 3 \mathrm{H}), 3.43(\mathrm{~s}, 3 \mathrm{H}), 1.58-2.04(\mathrm{~m}, 4 \mathrm{H}) ;{ }^{13} \mathrm{C}$ NMR for only major isomer $\left(400 \mathrm{MHz}, \mathrm{CDCl}_{3}\right) \delta 159.6,156.6,140.2,132.7,129.9,120.1,106.7,101.7,96.0$, 94.8, 74.8, 72.2, 66.4, 64.3, 56.2, 55.3, 45.7, 27.5, 25.8; HRMS (ESI-TOF) m/z: [M+Na] ${ }^{+} \mathrm{Calcd}$ for $\mathrm{C}_{20} \mathrm{H}_{30} \mathrm{O}_{8} \mathrm{Na} 421.1838$; Found 421.1831 .

(S)-3-((1S,2R)-1,3-dihydroxy-2-(4-methoxy-2-(methoxymethoxy)phenyl)propyl)-4(methoxymethoxy)cyclohex-2-en-1-one (26)<smiles>COc1ccc([C@H](CO)[C@H](O)C2=CC(O)CCC2OC)c(OC)c1</smiles>

25

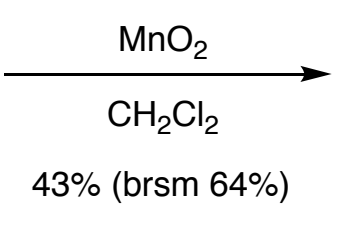

MOMO<smiles>COc1ccc([C@H](CO)[C@H](O)C2=CC(=O)CCC2OC)c(OC)c1</smiles>

26

To a solution of $25(17.0 \mathrm{mg}, 0.0427 \mathrm{mmol})$ in $\mathrm{CH}_{2} \mathrm{Cl}_{2}(0.5 \mathrm{ml})$ was added $\mathrm{MnO}_{2}(26.0 \mathrm{mg}, 0.299$ $\mathrm{mmol}$ ) at room temperature, and the mixture was stirred for $4 \mathrm{~h}$ at the same temperature. The 
insoluble material was removed by filtration and the filtrate was concentrated in vacuo. The resulting residue was purified by silica gel column chromatography $\left(\mathrm{CHCl}_{3}-\mathrm{MeOH}, 93: 7\right)$ to afford recovered $25(5.60 \mathrm{mg})$ and $\mathbf{2 6}$ (7.30 $\mathrm{mg}, 43 \%$ (brsm 64\%)) as colorless oil.

IR (neat) 3433, 2936, 1667, 1610, 1584, 1507, 1443, 1284, 1217, 1152, 1031, $922 \mathrm{~cm}^{-1},{ }^{1} \mathrm{H}$ NMR $\left(\mathrm{C}_{6} \mathrm{D}_{6}, 400 \mathrm{MHz}\right) \delta 7.55(\mathrm{~d}, 1 \mathrm{H}, J=8.0 \mathrm{~Hz}), 6.88(\mathrm{~d}, 1 \mathrm{H}, J=2.4 \mathrm{~Hz}), 6.50(\mathrm{dd}, 1 \mathrm{H}, J=2.4 \mathrm{~Hz}$, $8.0 \mathrm{~Hz}), 6.09$ (s, 1H), 4.86-4.91 (m, 1H), $4.80(\mathrm{~d}, 1 \mathrm{H}, J=6.4 \mathrm{~Hz}), 4.77$ (d, 1H, $J=6.4 \mathrm{~Hz}), 4.35$ $(\mathrm{d}, 1 \mathrm{H}, J=7.2 \mathrm{~Hz}), 4.29$ (d, 1H, $J=7.2 \mathrm{~Hz}), 4.06-4.11(\mathrm{~m}, 1 \mathrm{H}), 3.95-4.03(\mathrm{~m}, 1 \mathrm{H}), 3.70-3.83(\mathrm{~m}$, 2H), $3.48(\mathrm{~d}, 1 \mathrm{H}, J=3.6 \mathrm{~Hz}), 3.32(\mathrm{~s}, 3 \mathrm{H}), 3.15(\mathrm{~s}, 3 \mathrm{H}), 2.99(\mathrm{~s}, 3 \mathrm{H}), 2.48-2.57(\mathrm{~m}, 1 \mathrm{H}), 1.92-$ $2.07(\mathrm{~m}, 1 \mathrm{H}), 1.64-1.72(\mathrm{~m}, 1 \mathrm{H}), 1.38-1.49(\mathrm{~m}, 1 \mathrm{H}) ;{ }^{13} \mathrm{C} \mathrm{NMR}\left(\mathrm{C}_{6} \mathrm{D}_{6}, 400 \mathrm{MHz}\right) \delta 197.3,162.6$, 160.3, 157.0, 131.2, 127.1, 119.8, 106.8, 101.8, 96.4, 95.1, 73.4, 71.7, 64.5, 55.9, 55.5, 54.8, 45.2, 33.6, 29.1; HRMS (ESI-TOF) m/z: [M+Na] $]^{+}$Calcd for $\mathrm{C}_{20} \mathrm{H}_{28} \mathrm{O}_{8} \mathrm{Na} 419.1682$; Found 419.1676.

(3R,4S,5R,10S)-4-hydroxy-3-(4-methoxy-2-(methoxymethoxy)phenyl)-10(methoxymethoxy)-1-oxaspiro[4.5]decan-7-one (27)<smiles>COc1ccc([C@H](CO)[C@H](O)C2=CC(=O)CCC2OC)c(OC)c1</smiles>

26

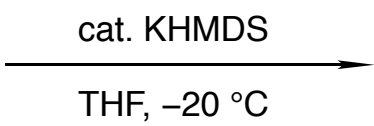

$62 \%$ (major isomer) $\mathrm{dr}=5: 1$<smiles>COc1ccc([C@H]2CO[C@]3(CC(=O)CCC3OC)C2O)c(OC)c1</smiles>

27

(single isomer after column separation)

To a solution of $\mathbf{2 6}(14.0 \mathrm{mg}, 0.0351 \mathrm{mmol})$ in THF $(0.5 \mathrm{ml})$ was added KHMDS ( $0.5 \mathrm{M}$ in toluene, $0.0100 \mathrm{ul}, 0.00500 \mathrm{mmol})$ at $-20{ }^{\circ} \mathrm{C}$ under Ar atmosphere, and the mixture was stirred for $1 \mathrm{~h}$ at the same temperature. The reaction mixture was quenched with sat. $\mathrm{NH}_{4} \mathrm{Cl}$ aq., allowed to warm to room temperature, and extracted with AcOEt (twice). The combined organic layers were washed with brine, dried over $\mathrm{MgSO}_{4}$, and concentrated in vacuo. The resulting residue was purified by silica gel column chromatography (hexane-AcOEt, 3:7) to afford 27 (8.70 $\mathrm{mg}$, single isomer, $62 \%$ ) as colorless oil.

IR (neat) 3466, 2952, 1714, 1612, 1585, 1508, 1291, 1217, 1154, 1127, 1075, $1019 \mathrm{~cm}^{-1},{ }^{1} \mathrm{H}$ NMR $\left(\mathrm{C}_{6} \mathrm{D}_{6}, 400 \mathrm{MHz}\right) \delta 7.22(\mathrm{~d}, 1 \mathrm{H}, J=8.4 \mathrm{~Hz}), 6.87(\mathrm{~d}, 1 \mathrm{H}, J=2.4 \mathrm{~Hz}), 6.43(\mathrm{dd}, 1 \mathrm{H}, J=2.4 \mathrm{~Hz}$, $8.4 \mathrm{~Hz}), 4.81(\mathrm{~d}, 1 \mathrm{H}, J=6.8 \mathrm{~Hz}), 4.79(\mathrm{~d}, 1 \mathrm{H}, J=6.8 \mathrm{~Hz}), 4.25-4.34(\mathrm{~m}, 2 \mathrm{H}), 4.28$ (d, 1H, $J=$ $6.0 \mathrm{~Hz}), 4.25(\mathrm{~d}, 1 \mathrm{H}, J=6.0 \mathrm{~Hz}), 4.09-4.12(\mathrm{~m}, 1 \mathrm{H}), 3.99$ (dd, $1 \mathrm{H}, J=7.6 \mathrm{~Hz}, 8.8 \mathrm{~Hz}), 3.79-3.84$ 
(m, 1H), $3.61(\mathrm{~d}, 1 \mathrm{H}, J=3.2 \mathrm{~Hz}), 3.32(\mathrm{~s}, 3 \mathrm{H}), 3.10(\mathrm{~s}, 3 \mathrm{H}), 2.96(\mathrm{~s}, 3 \mathrm{H}), 2.75(\mathrm{~d}, 1 \mathrm{H}, J=14.0$ $\mathrm{Hz}), 2.63(\mathrm{~d}, 1 \mathrm{H}, J=14.0 \mathrm{~Hz}), 2.26-2.36(\mathrm{~m}, 1 \mathrm{H}), 2.10-2.24(\mathrm{~m}, 2 \mathrm{H}), 1.73-1.82(\mathrm{~m}, 1 \mathrm{H}) ;{ }^{13} \mathrm{C}$ $\operatorname{NMR}\left(\mathrm{C}_{6} \mathrm{D}_{6}, 400 \mathrm{MHz}\right) \delta 206.5,160.3,156.6,129.0,127.9,120.6,106.4,102.1,95.8,94.4,88.3$, 82.8, 74.6, 69.9, 55.7, 55.7, 54.9, 49.4, 46.7, 36.0, 25.9; HRMS (ESI-TOF) m/z: [M+Na] ${ }^{+} \mathrm{Calcd}$ for $\mathrm{C}_{20} \mathrm{H}_{28} \mathrm{O}_{8} \mathrm{Na} 419.1682$; Found 419.1677 .

(3R,4S,5S,6S)-3-(4-methoxy-2-(methoxymethoxy)phenyl)-6-(methoxymethoxy)-9-oxo1-oxaspiro[4.5]decan-4-yl methanesulfonate (S3)<smiles>COc1ccc(C2COC3(CC(=O)CCC3OC)C2O)c(OC)c1</smiles>

27

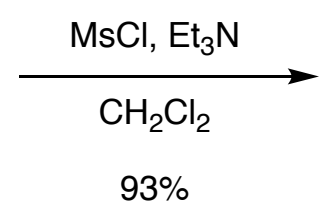

$93 \%$<smiles>COc1ccc([C@H]2COC3(CC(=O)CCC3OC)[C@H]2OC)c(OC)c1</smiles>

S3

To a solution of $27(5.00 \mathrm{mg}, 0.0126 \mathrm{mmol})$ in $\mathrm{CH}_{2} \mathrm{Cl}_{2}(0.5 \mathrm{ml})$ was added $\mathrm{Et}_{3} \mathrm{~N}$ (50.0 ul, 0.359 $\mathrm{mmol})$ and $\mathrm{MsCl}(25.0 \mathrm{ul}, 0.323 \mathrm{mmol})$ at $0{ }^{\circ} \mathrm{C}$ under $\mathrm{Ar}$ atmosphere, and the mixture was stirred for $1 \mathrm{~h}$ at room temperature. The reaction mixture was quenched with $\mathrm{H}_{2} \mathrm{O}$, and extracted with $\mathrm{CHCl}_{3}$ (twice). The combined organic layers were washed with brine, dried over $\mathrm{MgSO}_{4}$, and concentrated in vacuo. The resulting residue was purified by silica gel column chromatography (hexane-AcOEt, 1:1) to afford $\mathbf{S 3}$ (5.60 mg, 93\%) as colorless oil.

IR (neat) 2936, 1715, 1613, 1508, 1354, 1175, 1128, 1043, 996, 930, $855 \mathrm{~cm}^{-1} ;{ }^{1} \mathrm{H}$ NMR $\left(\mathrm{C}_{6} \mathrm{D}_{6}\right.$, $400 \mathrm{MHz}) \delta 6.89(\mathrm{~d}, 1 \mathrm{H}, J=8.4 \mathrm{~Hz}), 6.80(\mathrm{~d}, 1 \mathrm{H}, J=2.4 \mathrm{~Hz}), 6.32(\mathrm{dd}, 1 \mathrm{H}, J=2.4 \mathrm{~Hz}, 8.4 \mathrm{~Hz})$, $5.28(\mathrm{~d}, 1 \mathrm{H}, J=5.2 \mathrm{~Hz}), 5.11(\mathrm{~d}, 1 \mathrm{H}, J=7.6 \mathrm{~Hz}), 4.89$ (d, 1H, $J=7.6 \mathrm{~Hz}), 4.72(\mathrm{~s}, 2 \mathrm{H}), 3.83-$ $3.99(\mathrm{~m}, 3 \mathrm{H}), 3.42(\mathrm{td}, 1 \mathrm{H}, J=5.2 \mathrm{~Hz}, 8.0 \mathrm{~Hz}), 3.27$ (s, 3H), 3.12 (s, 3H), 3.08 (d, $1 \mathrm{H}, J=14.0$ $\mathrm{Hz}), 2.66(\mathrm{~d}, 1 \mathrm{H}, J=14.0 \mathrm{~Hz}), 2.52-2.63(\mathrm{~m}, 1 \mathrm{H}), 2.19-2.35(\mathrm{~m}, 3 \mathrm{H}), 2.15(\mathrm{~s}, 3 \mathrm{H}) ;{ }^{13} \mathrm{C} \mathrm{NMR}$ $\left(\mathrm{C}_{6} \mathrm{D}_{6}, 400 \mathrm{MHz}\right) \delta 206.1,161.0,156.2,130.2,117.0,106.6,101.8,99.2,94.1,89.4,87.8,75.4$, 68.8, 56.0, 55.9, 54.9, 48.8, 46.2, 38.3, 36.3, 30.6, 27.6; HRMS (ESI-TOF) m/z: [M+Na] ${ }^{+} \mathrm{Calcd}$ for $\mathrm{C}_{21} \mathrm{H}_{30} \mathrm{O}_{10} \mathrm{SNa}$ 497.1457; Found 497.1458.

(3R,4S,5S,6S)-6-hydroxy-3-(2-hydroxy-4-methoxyphenyl)-9-oxo-1-oxaspiro[4.5]decan4-yl methanesulfonate (28) 
<smiles>COc1ccc([C@H]2CO[C@]3(CC(=O)CCC3OC)C2OC)c(OC)c1</smiles>

S3

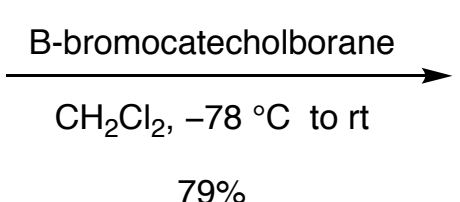

$79 \%$

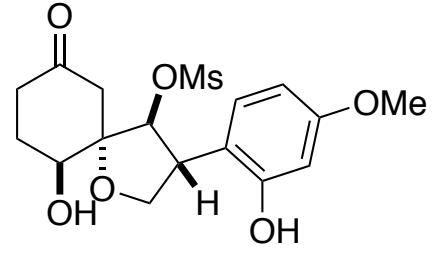

28

To a solution of $\mathbf{S 3}(3.00 \mathrm{mg}, 0.00632 \mathrm{mmol})$ in $\mathrm{CH}_{2} \mathrm{Cl}_{2}(0.5 \mathrm{ml})$ was added Bbromocatecholborane $(15.0 \mathrm{mg}, 0.0754 \mathrm{mmol})$ at $-78^{\circ} \mathrm{C}$ under $\mathrm{Ar}$ atmosphere, and the mixture was stirred for $2 \mathrm{~h}$ at the same temperature. The reaction mixture was allowed to warm to room temperature over $15 \mathrm{~min}$, quenched with sat. $\mathrm{NaHCO}_{3}$ aq., and extracted with $\mathrm{AcOEt}$ (three times). The combined organic layers were washed with brine, dried over $\mathrm{MgSO}_{4}$, and concentrated in vacuo. The resulting residue was purified by preparative TLC (hexane-AcOEt, 1:2) to afford 28 (1.90 mg, 79\%) as colorless oil.

IR (neat) 3408, 2922, 2851, 1709, 1618, 1522, 1444, 1349, 1172, 1116, 1054, $959 \mathrm{~cm}^{-1}$; ${ }^{1} \mathrm{H}$ NMR $\left(\mathrm{CD}_{3} \mathrm{OD}, 400 \mathrm{MHz}\right) \delta 7.11(\mathrm{~d}, 1 \mathrm{H}, J=8.0 \mathrm{~Hz}), 6.39-6.45(\mathrm{~m}, 2 \mathrm{H}), 5.28(\mathrm{~d}, 1 \mathrm{H}, J=5.2 \mathrm{~Hz}), 4.05-$ $4.18(\mathrm{~m}, 3 \mathrm{H}), 3.74(\mathrm{~s}, 3 \mathrm{H}), 3.64-3.73(\mathrm{~m}, 1 \mathrm{H}), 3.02$ (d, 1H, $J=14.0 \mathrm{~Hz}), 2.93(\mathrm{~m}, 3 \mathrm{H}), 2.65-2.77$ $(\mathrm{m}, 1 \mathrm{H}), 2.49(\mathrm{~d}, 1 \mathrm{H}, J=14.0 \mathrm{~Hz}), 1.97-2.26(\mathrm{~m}, 3 \mathrm{H}) ;{ }^{13} \mathrm{C} \mathrm{NMR}\left(\mathrm{CD}_{3} \mathrm{OD}, 400 \mathrm{MHz}\right) \delta 212.1$, 161.9, 157.6, 131.3, 116.1, 106.0, 102.7, 90.2, 89.5, 69.7, 66.9, 55.6, 50.2, 46.0, 38.7, 36.4, 30.2; HRMS (ESI-TOF) m/z: [M+Na] $]^{+}$Calcd for $\mathrm{C}_{17} \mathrm{H}_{22} \mathrm{O}_{8} \mathrm{SNa}$ 409.0933; Found 409.0925.

( $\left.1 S, 2 S, 3 \mathrm{a}^{\prime} R, 8 \mathrm{~b}^{\prime} R\right)$-2-hydroxy-6'-methoxy-3a',8b'-dihydro-1' $H$-spiro[cyclohexane-1,3'furo[3,4-b] benzofuran]-5-one (stachyodin A, racemic 1)<smiles>COc1ccc([C@H]2COC3(CC(=O)CCC3O)C2O)c(O)c1</smiles>

28

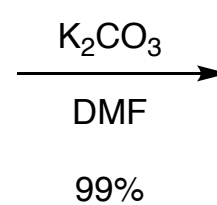<smiles>COc1ccc2c(c1)OC1(COC3CCC(=O)CC31)C2</smiles>

stachyodin A (racemic 1)

To a solution of $\mathbf{2 8}(2.90 \mathrm{mg}, 0.00750 \mathrm{mmol})$ in DMF $(0.2 \mathrm{ml})$ was added powder $\mathrm{K}_{2} \mathrm{CO}_{3}(18.0$ $\mathrm{mg}, 0.130 \mathrm{mmol}$ ) at $0{ }^{\circ} \mathrm{C}$ under $\mathrm{Ar}$ atmosphere, and the mixture was stirred for $5 \mathrm{~h}$ at room temperature. The reaction mixture was quenched with sat. $\mathrm{NH}_{4} \mathrm{Cl}$ aq., and extracted with $\mathrm{AcOEt}$ (twice). The combined organic layers were washed with brine, dried over $\mathrm{MgSO}_{4}$, and concentrated in vacuo. The resulting residue was purified by preparative TLC (hexane-AcOEt, 
1:2) to afford racemic 1 (2.20 $\mathrm{mg}, 99 \%)$ as colorless oil.

IR (neat) 3583, 2919, 1708, 1622, 1598, 1498, 1446, 1196, 1147, 1116, $1067 \mathrm{~cm}^{-1} ;{ }^{1} \mathrm{H}$ NMR $\left(\mathrm{CD}_{3} \mathrm{OD}, 400 \mathrm{MHz}\right) \delta 7.06(\mathrm{~d}, 1 \mathrm{H}, J=8.4 \mathrm{~Hz}), 6.45(\mathrm{dd}, 1 \mathrm{H}, J=2.4 \mathrm{~Hz}, 8.4 \mathrm{~Hz}), 6.35(\mathrm{~d}, 1 \mathrm{H}, J$ $=2.4 \mathrm{~Hz}), 5.43(\mathrm{~d}, 1 \mathrm{H}, J=8.4 \mathrm{~Hz}), 4.07-4.15(\mathrm{~m}, 2 \mathrm{H}), 3.85-3.89(\mathrm{~m}, 2 \mathrm{H}), 3.74(\mathrm{~s}, 3 \mathrm{H}), 2.94(\mathrm{~d}$, $1 \mathrm{H}, J=14.8 \mathrm{~Hz}), 2.65-2.74(\mathrm{~m}, 1 \mathrm{H}), 2.16(\mathrm{~d}, 1 \mathrm{H}, J=14.8 \mathrm{~Hz}), 2.12-2.24(\mathrm{~m}, 2 \mathrm{H}), 1.93-2.02(\mathrm{~m}$, $1 \mathrm{H}) ;{ }^{13} \mathrm{C}$ NMR $\left(\mathrm{CD}_{3} \mathrm{OD}, 400 \mathrm{MHz}\right) \delta 212.2,162.5,162.4,125.6,122.3,107.9,96.4,92.2,90.7$, 73.9, 66.9, 55.9, 48.4, 44.0, 36.8, 29.3; HRMS (ESI-TOF) m/z: [M+Na] Calcd for $\mathrm{C}_{16} \mathrm{H}_{18} \mathrm{O}_{5} \mathrm{Na}$ 313.1052; Found 313.1044. 


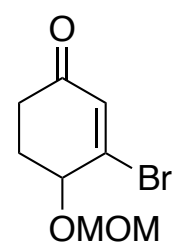

15

(400 MHz, $\mathrm{CDCl}_{3}$ )

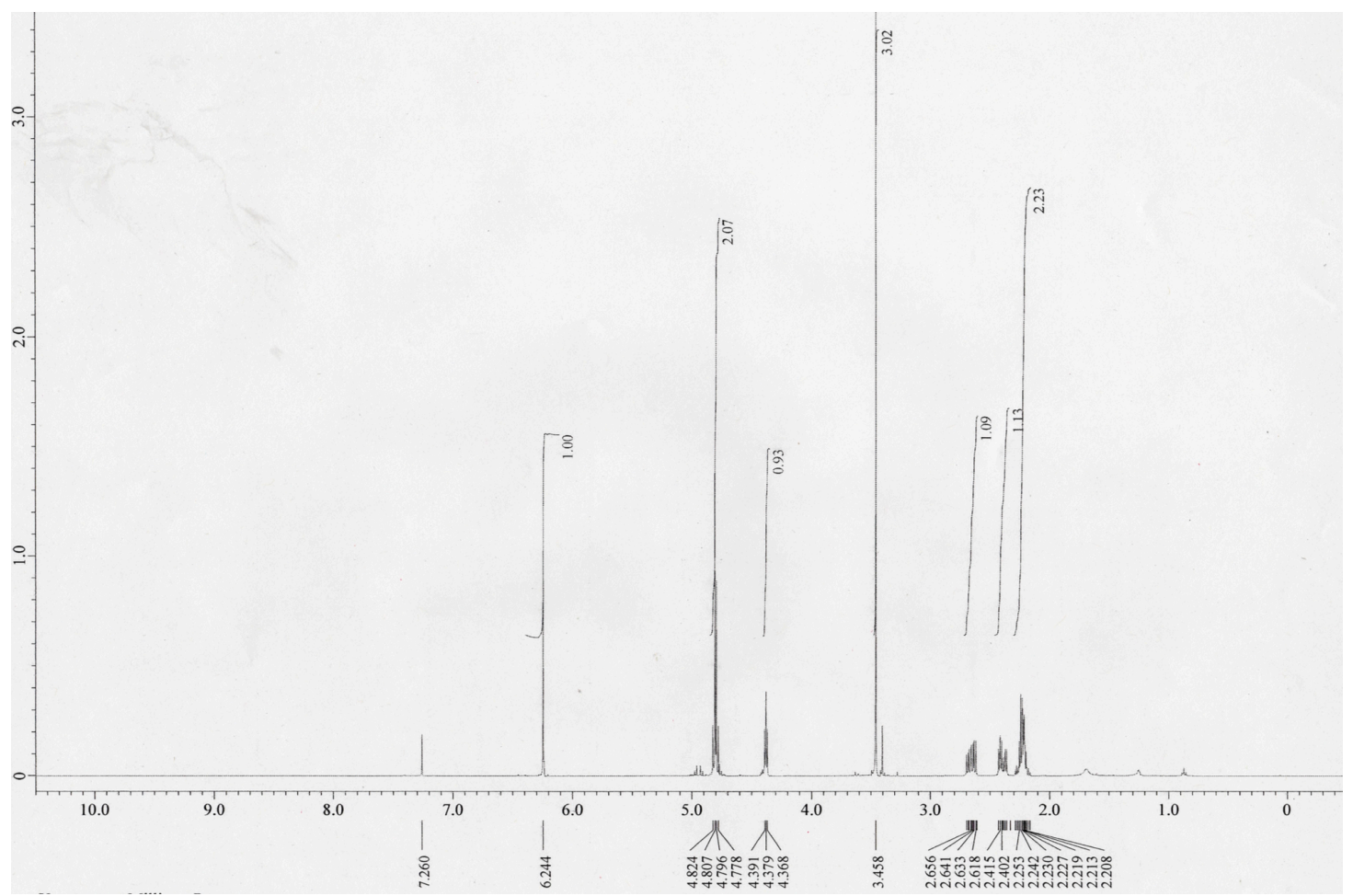


<smiles>COC1CCC(=O)C=C1Br</smiles>

\section{5}

(400 MHz, $\mathrm{CDCl}_{3}$ )

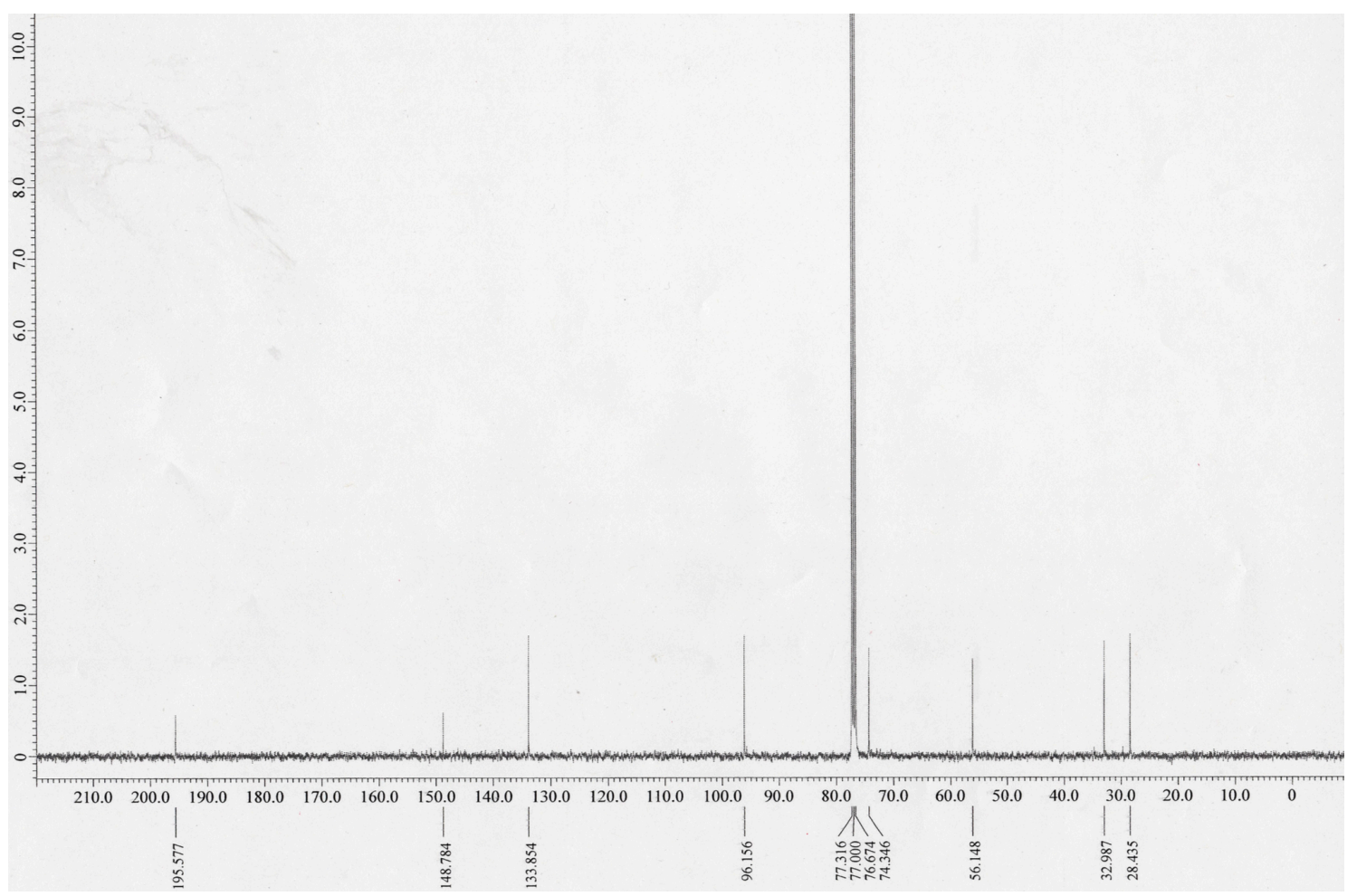




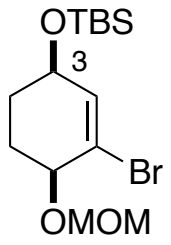

16

(5:1 mixture at $\mathrm{C} 3$ )

(400 MHz, $\mathrm{CDCl}_{3}$ )

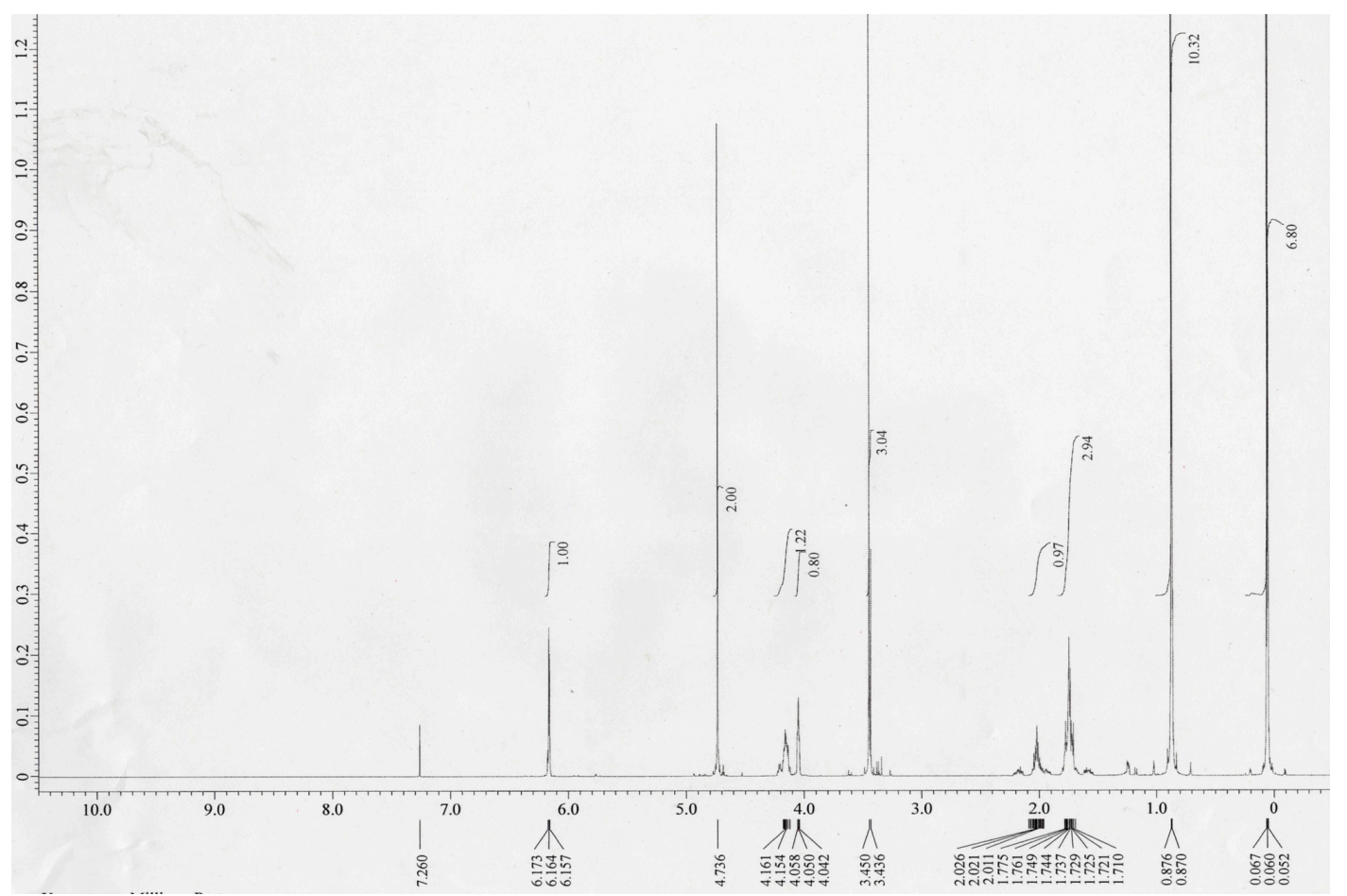




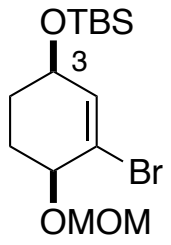

16

(5:1 mixture at $\mathrm{C} 3$ )

$\left(400 \mathrm{MHz}, \mathrm{CDCl}_{3}\right.$ )

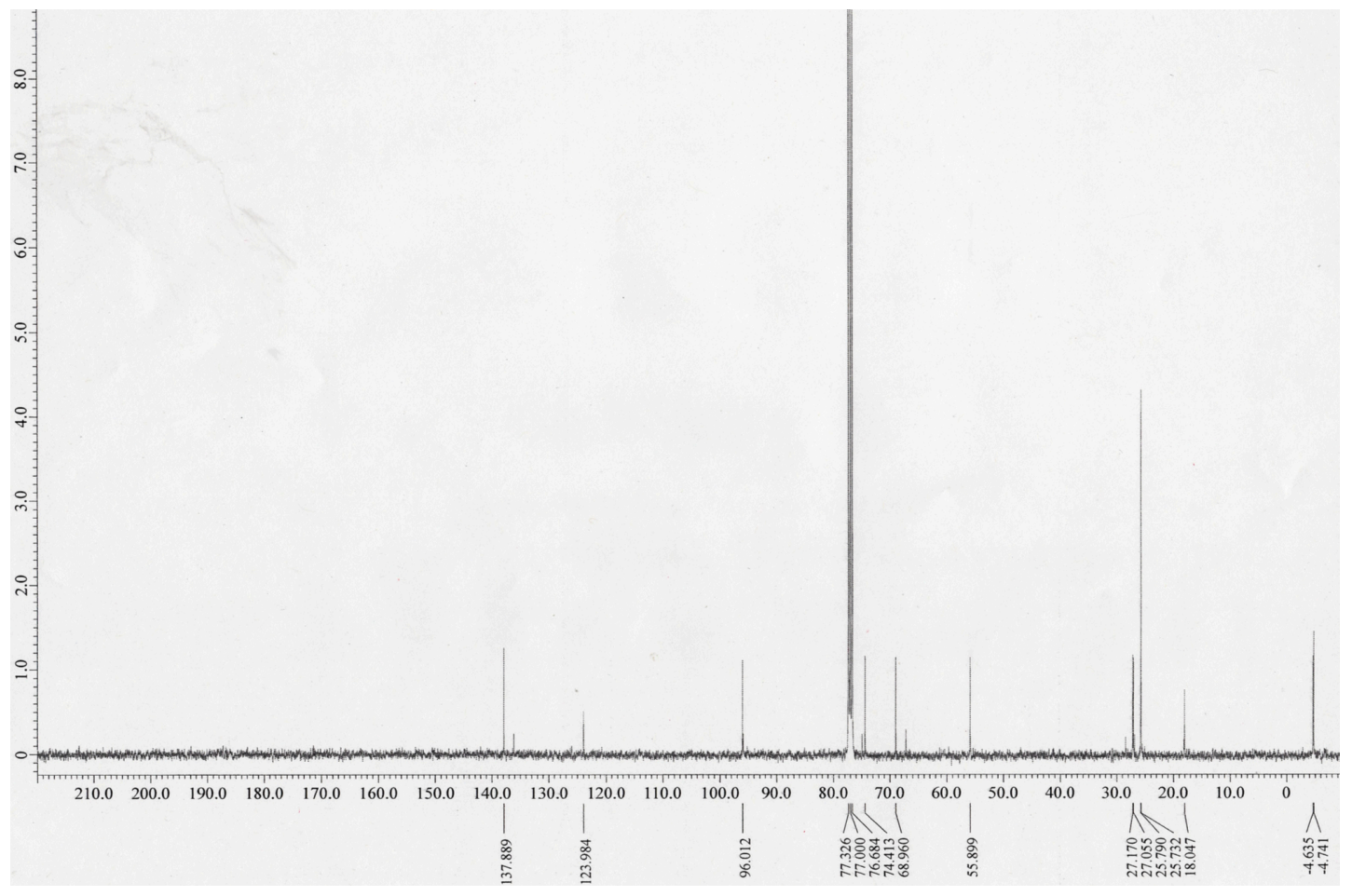


<smiles>COC1CCC(OC(=O)c2ccc(Br)cc2)C=C1Br</smiles>

S2 (mixture at $\mathrm{C} 3$ )

$\left(400 \mathrm{MHz}, \mathrm{CDCl}_{3}\right.$ )

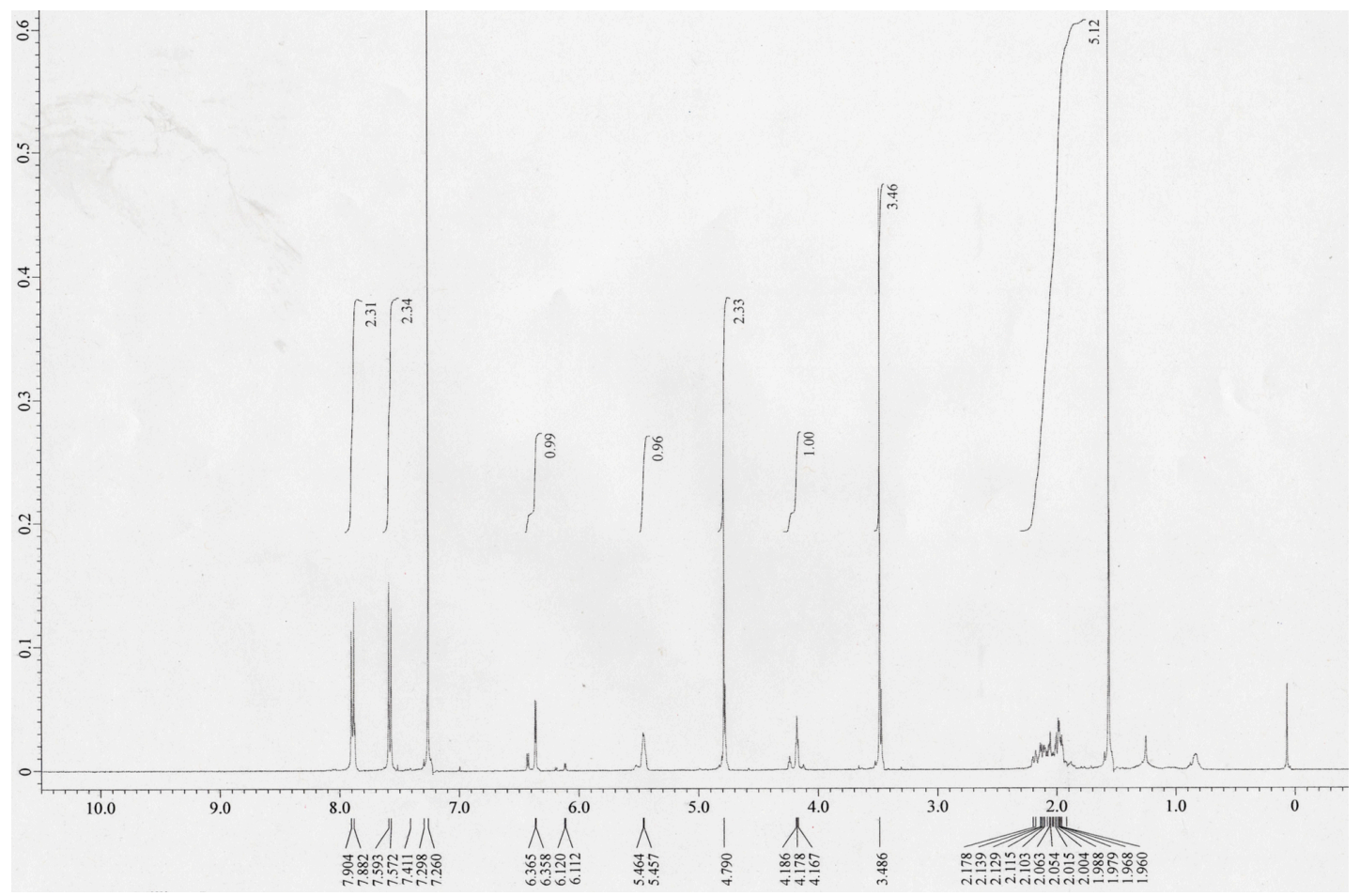


<smiles>COC1CCC(OC(=O)c2ccc(Br)cc2)C=C1Br</smiles>

S2 (mixture at $\mathrm{C} 3$ ) $\left(400 \mathrm{MHz}, \mathrm{CDCl}_{3}\right.$ )

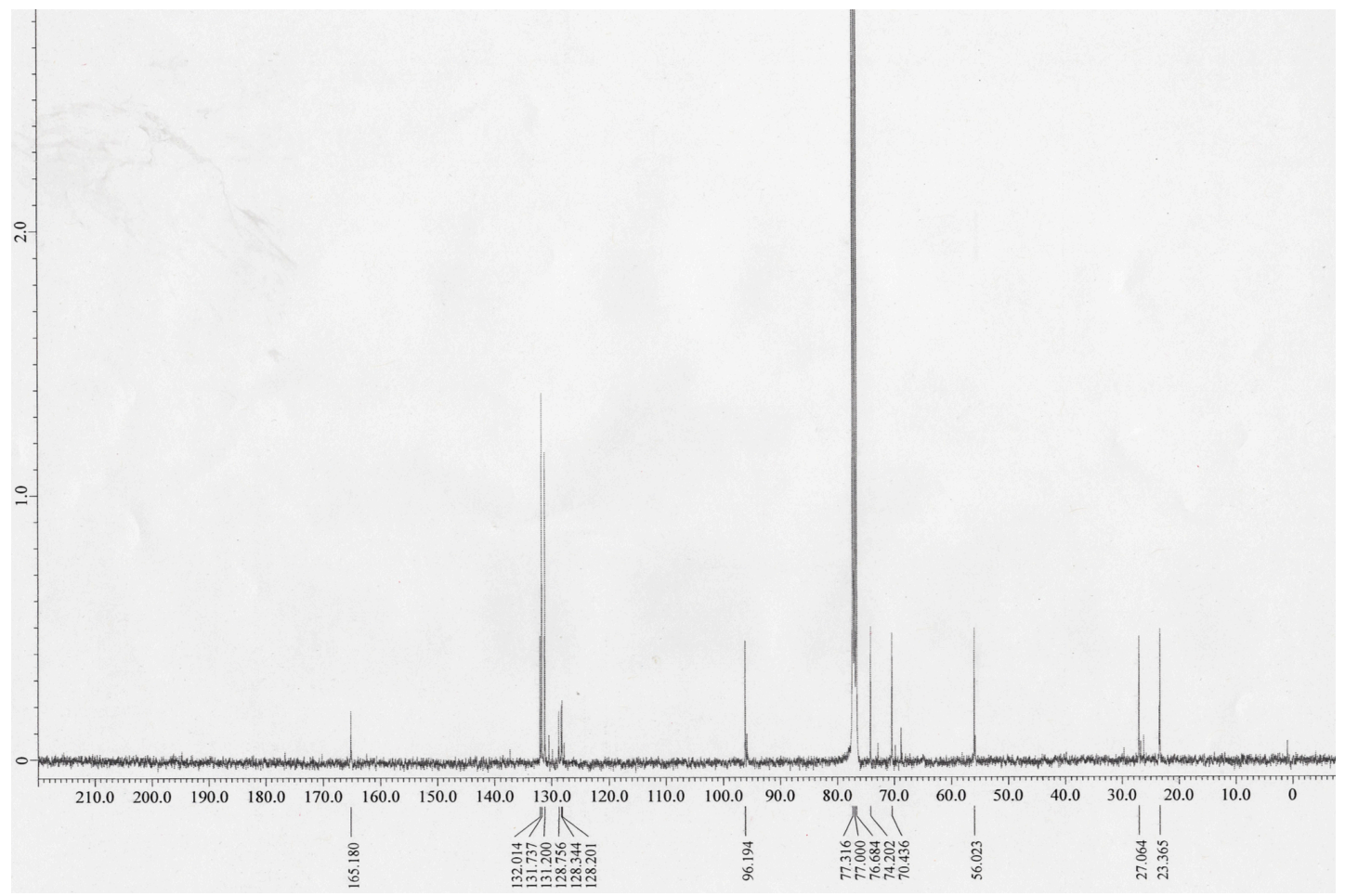




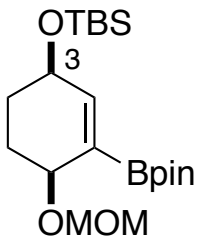

17

(5:1 mixture at $\mathrm{C} 3$ )

(400 MHz, $\mathrm{CDCl}_{3}$ )

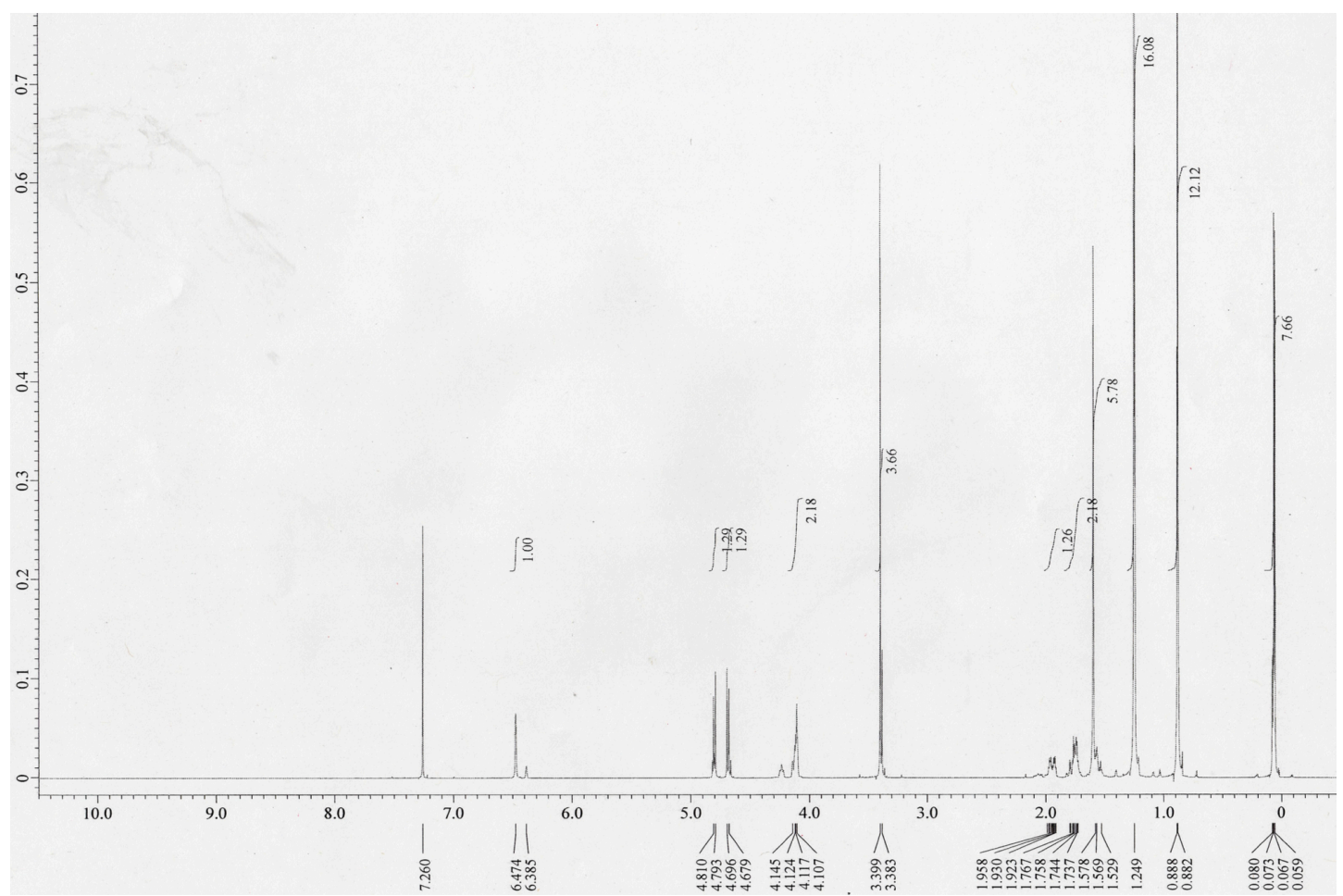




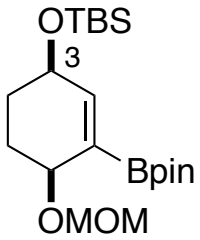

17

(5:1 mixture at $\mathrm{C} 3$ )

(400 MHz, $\mathrm{CDCl}_{3}$ )

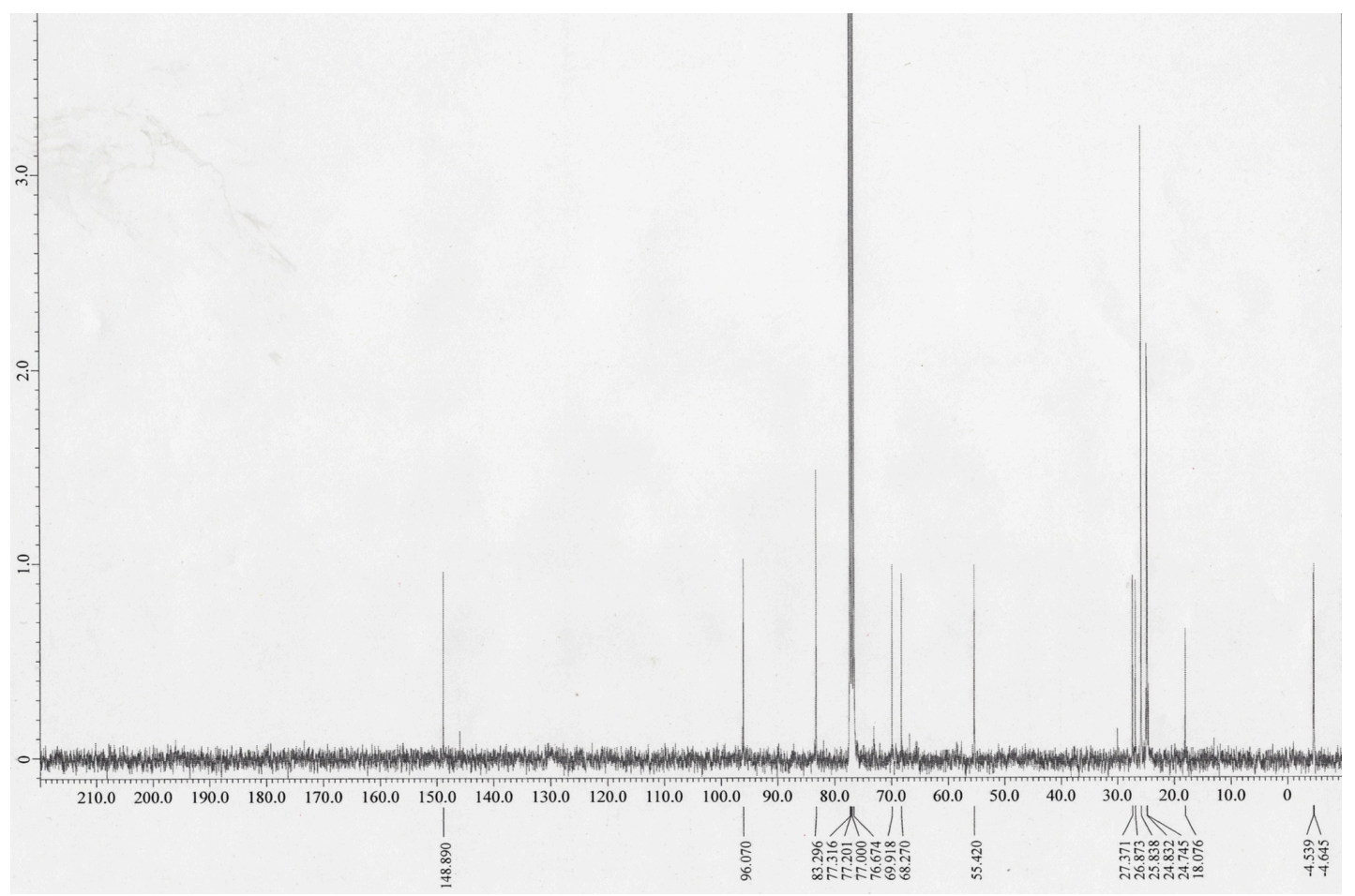




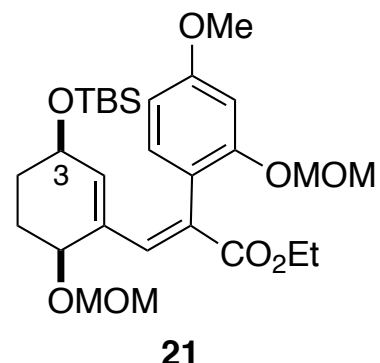

(5:1 mixture at $\mathrm{C} 3)$

$\left(400 \mathrm{MHz}, \mathrm{CDCl}_{3}\right.$ )

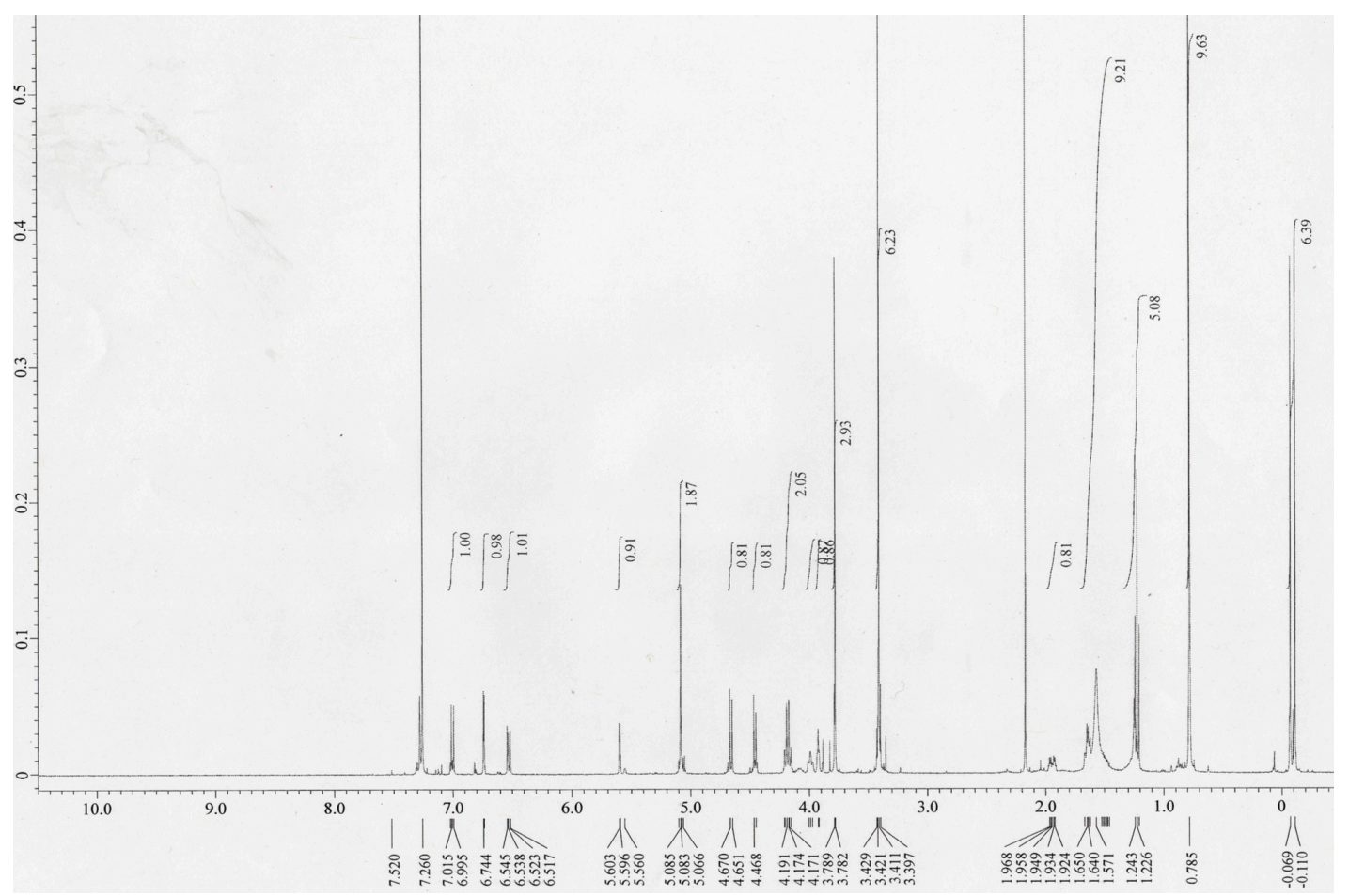




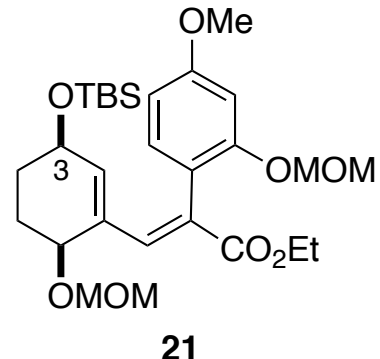

(5:1 mixture at $\mathrm{C} 3$ )

(400 MHz, $\mathrm{CDCl}_{3}$ )

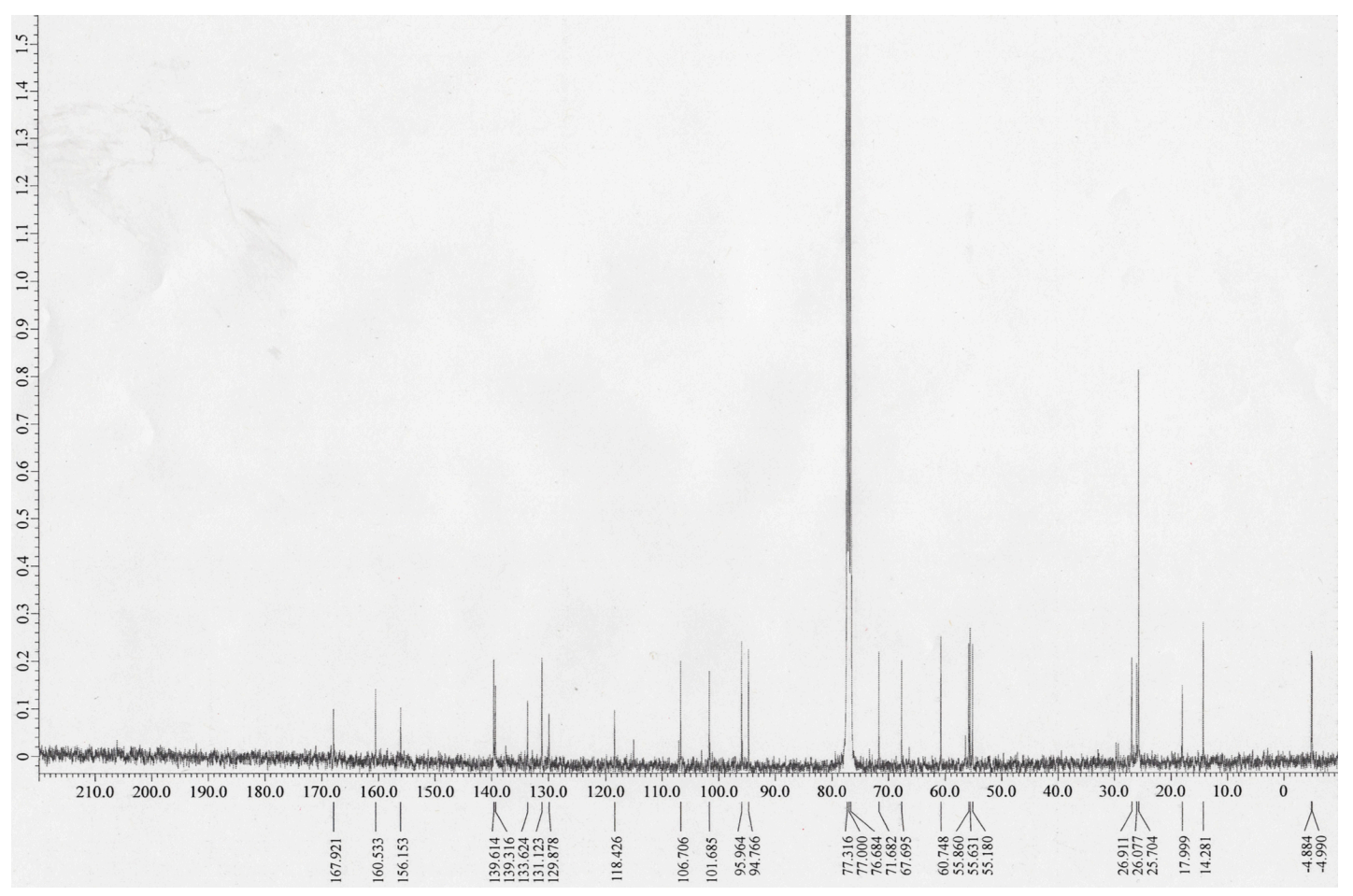




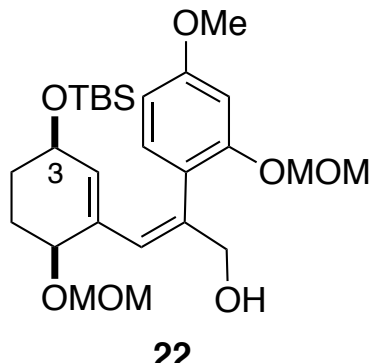

(5:1 mixture at $\mathrm{C} 3$ )

$\left(400 \mathrm{MHz}, \mathrm{C}_{6} \mathrm{D}_{6}\right.$ )

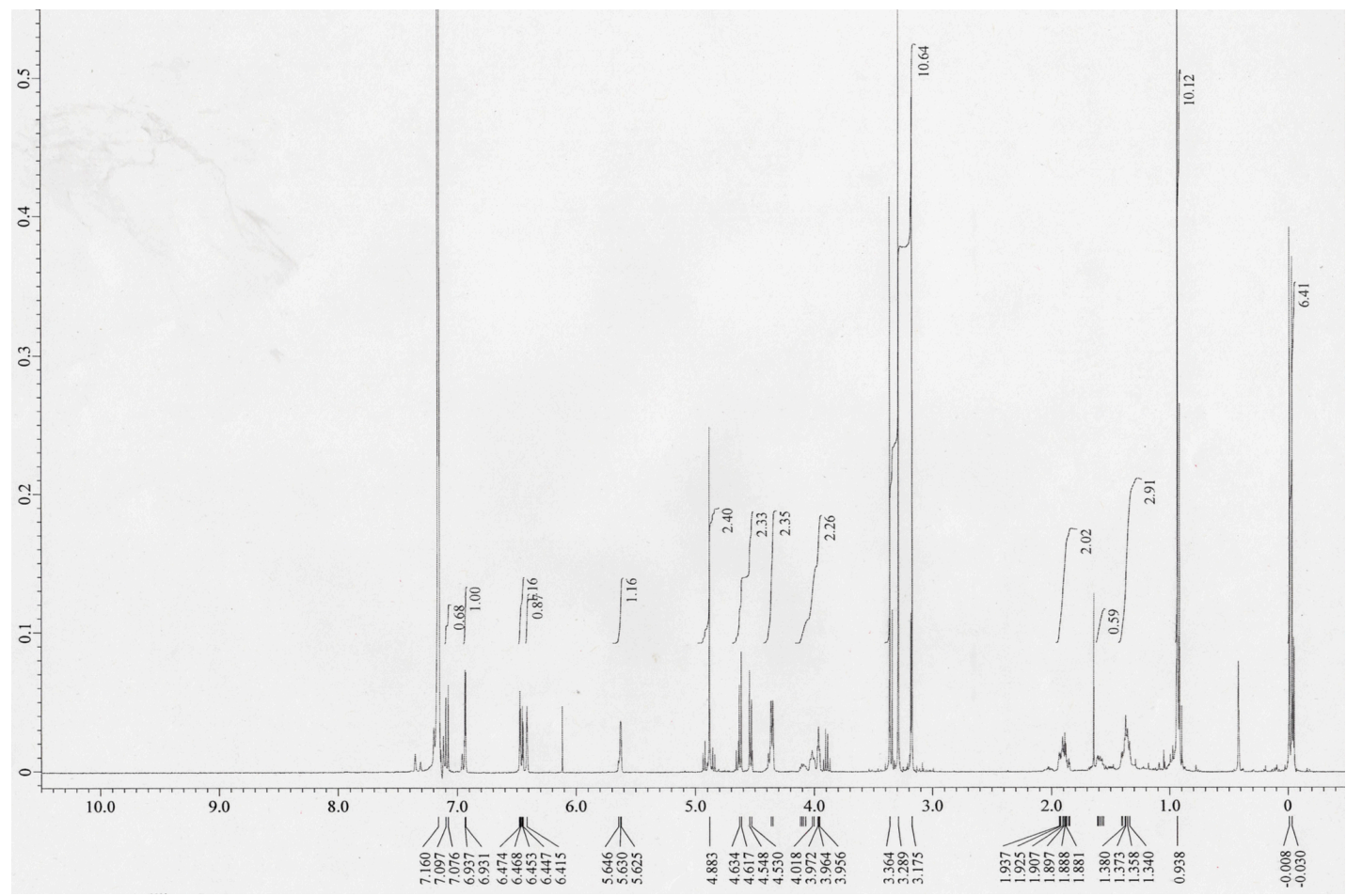




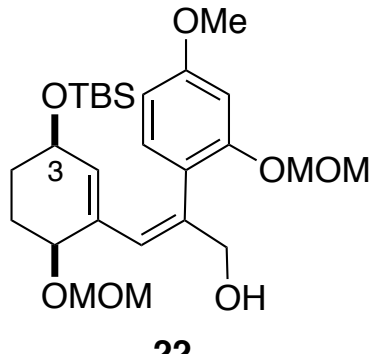

22

(5:1 mixture at $\mathrm{C} 3$ )

$\left(400 \mathrm{MHz}, \mathrm{C}_{6} \mathrm{D}_{6}\right)$

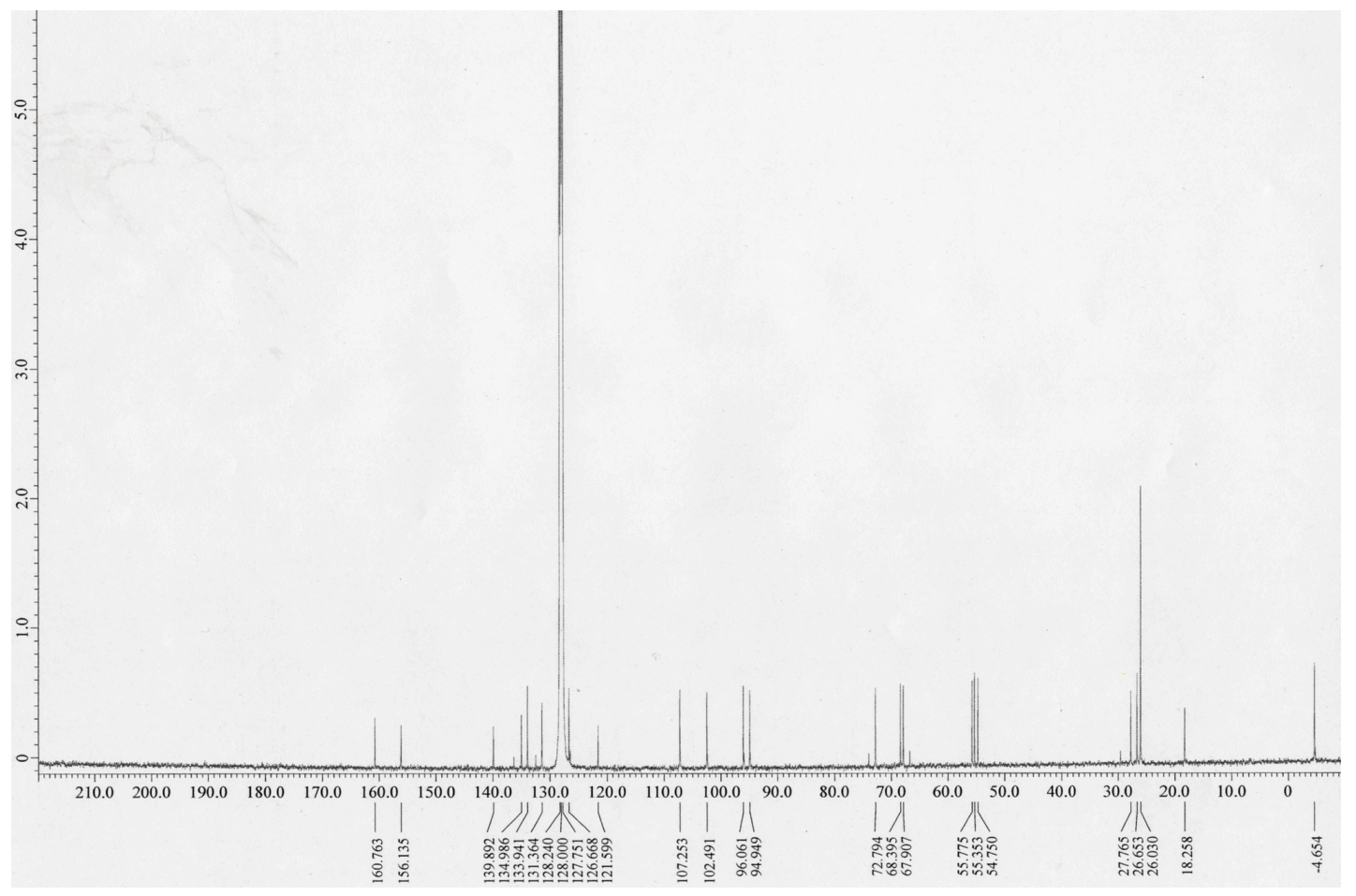




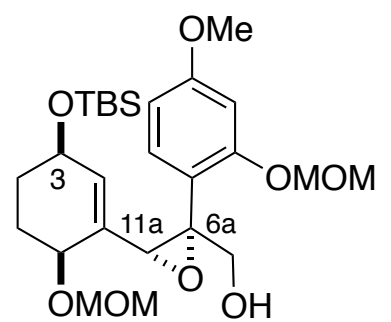

23' (single isomer)

$\left(400 \mathrm{MHz}, \mathrm{C}_{6} \mathrm{D}_{6}\right)$

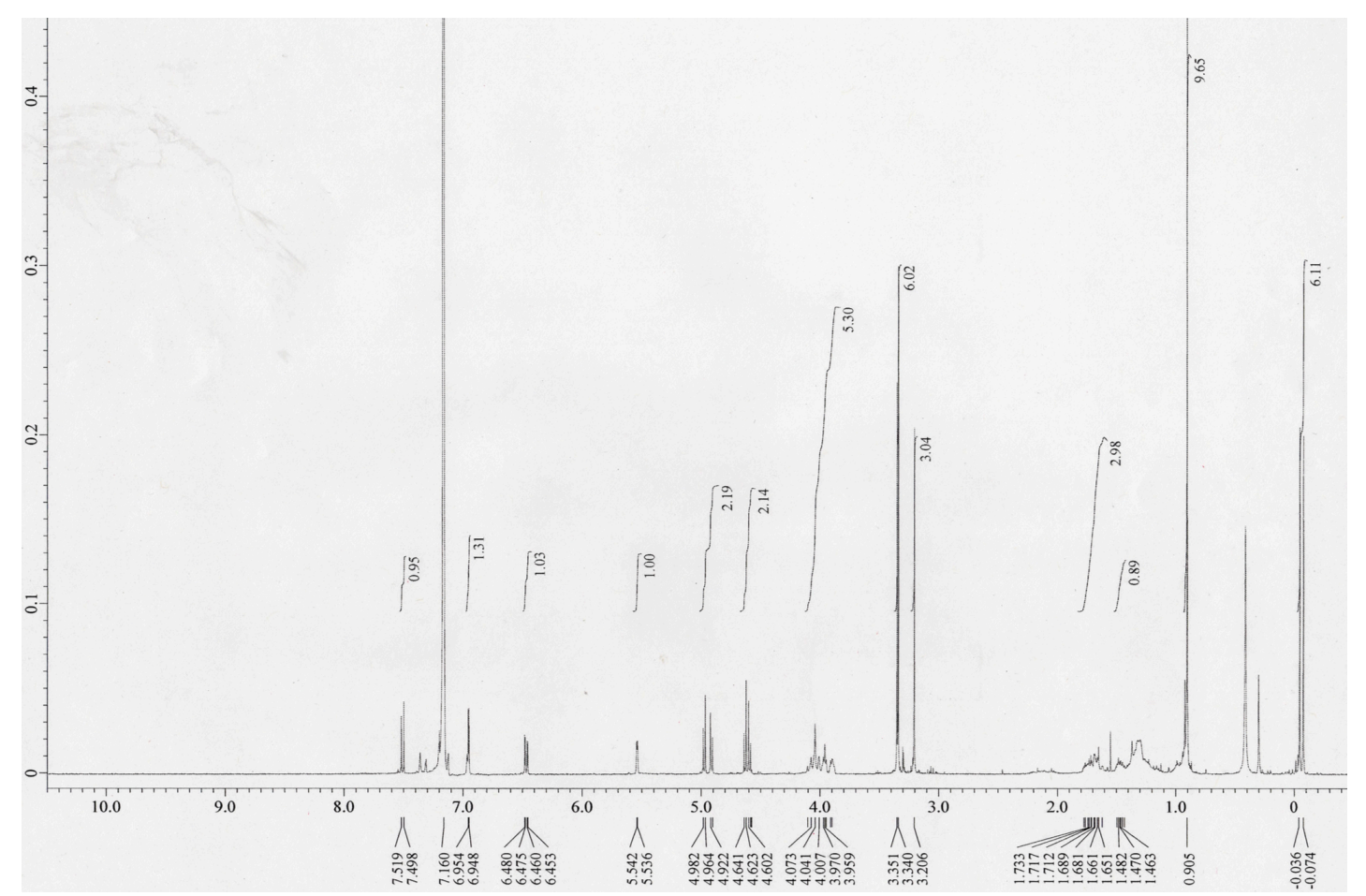




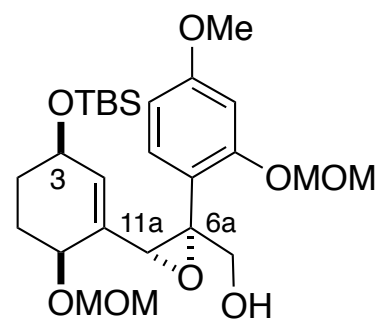

23 (5:1 mixture at C3) $\left(400 \mathrm{MHz}, \mathrm{C}_{6} \mathrm{D}_{6}\right.$ )

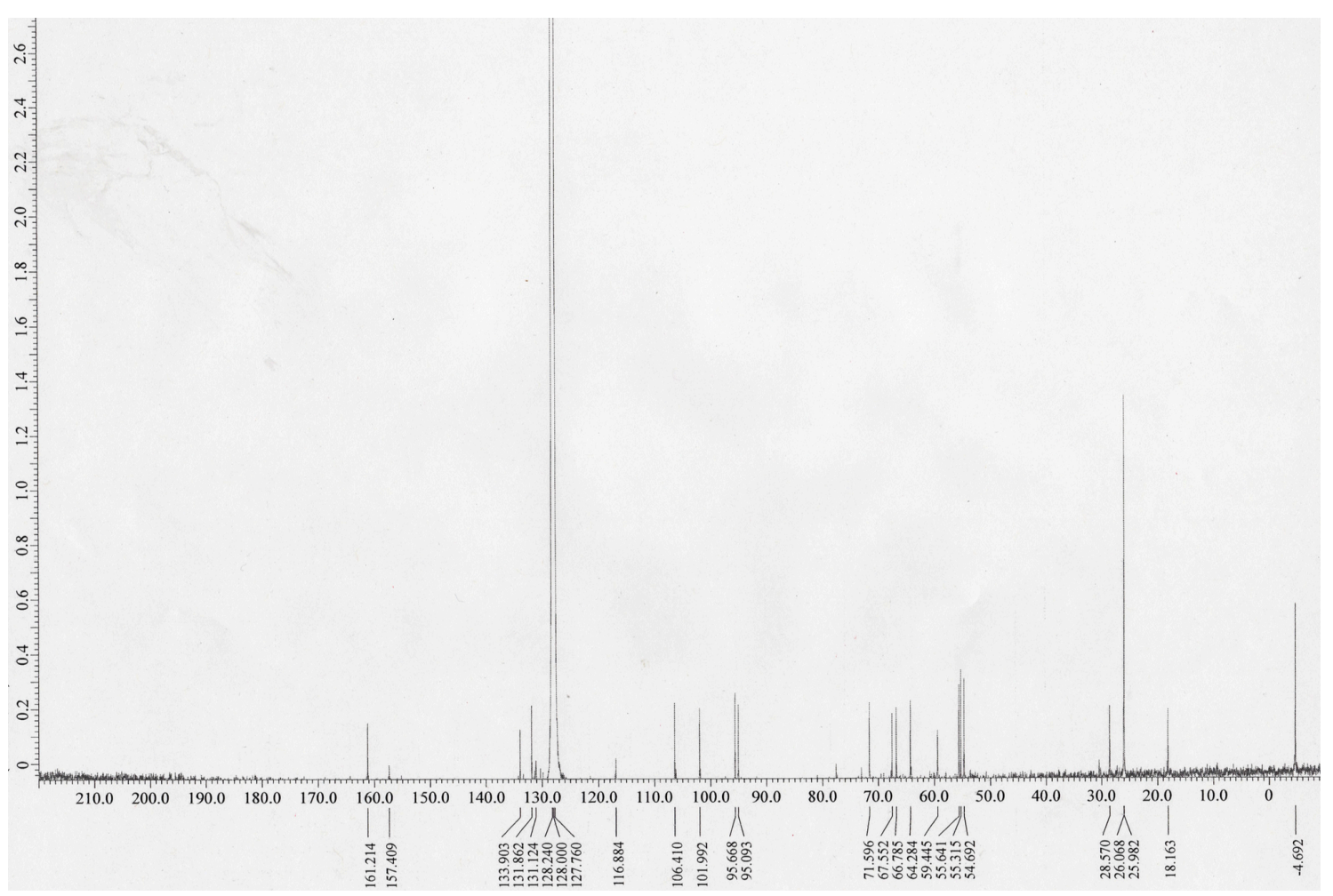




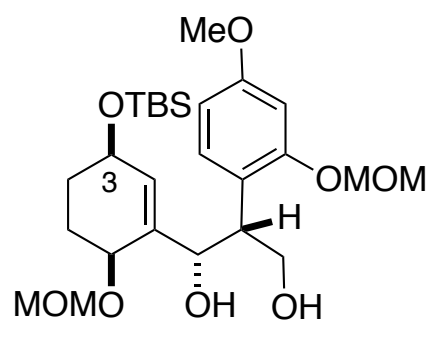

24

(5:1 mixture at $\mathrm{C} 3$ )

$\left(400 \mathrm{MHz}, \mathrm{C}_{6} \mathrm{D}_{6}\right)$

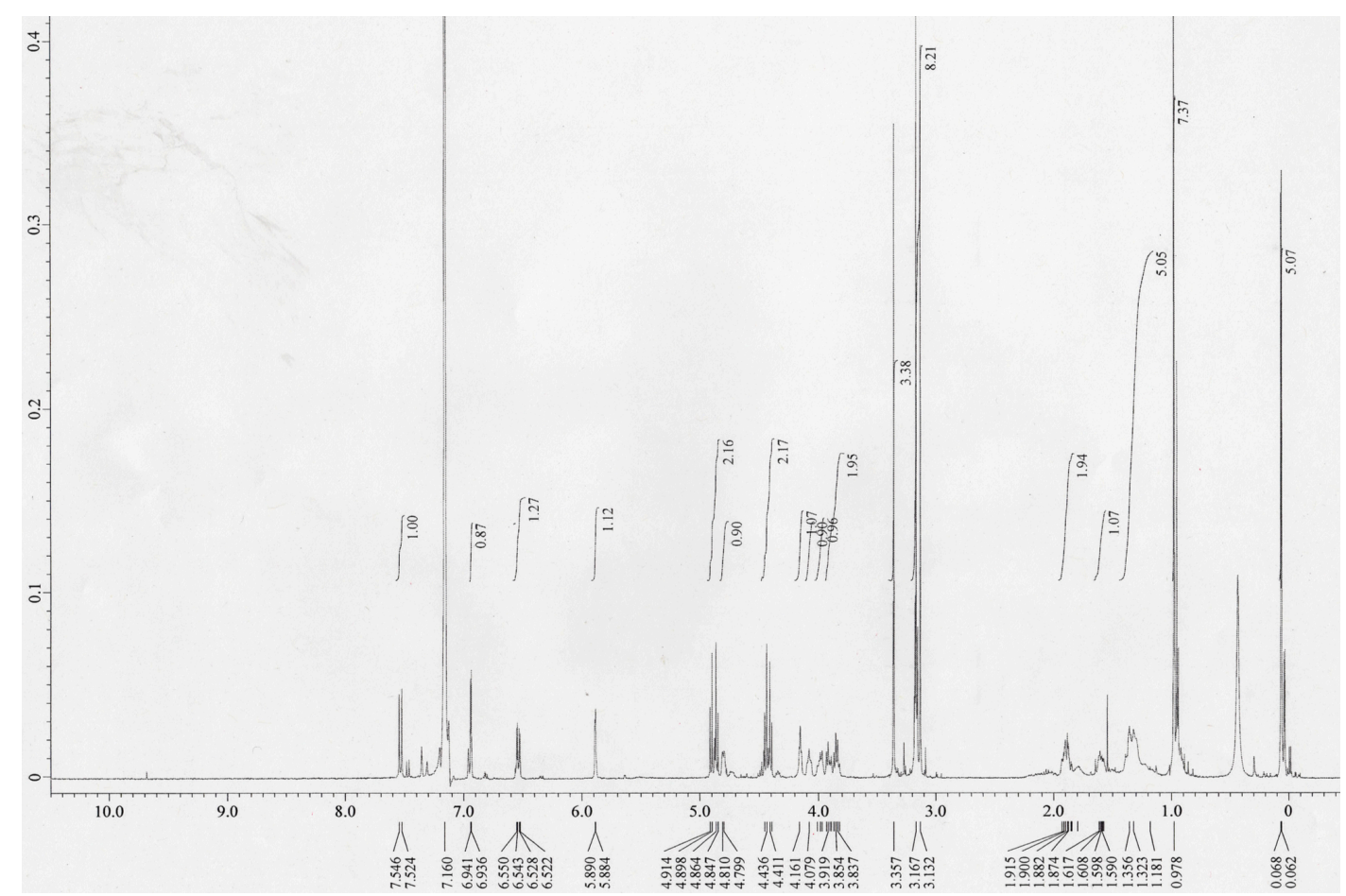




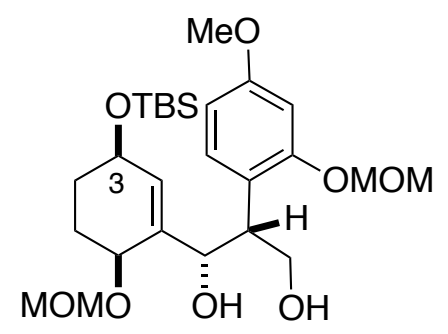

24

(5:1 mixture at $\mathrm{C} 3$ )

$\left(400 \mathrm{MHz}, \mathrm{C}_{6} \mathrm{D}_{6}\right)$

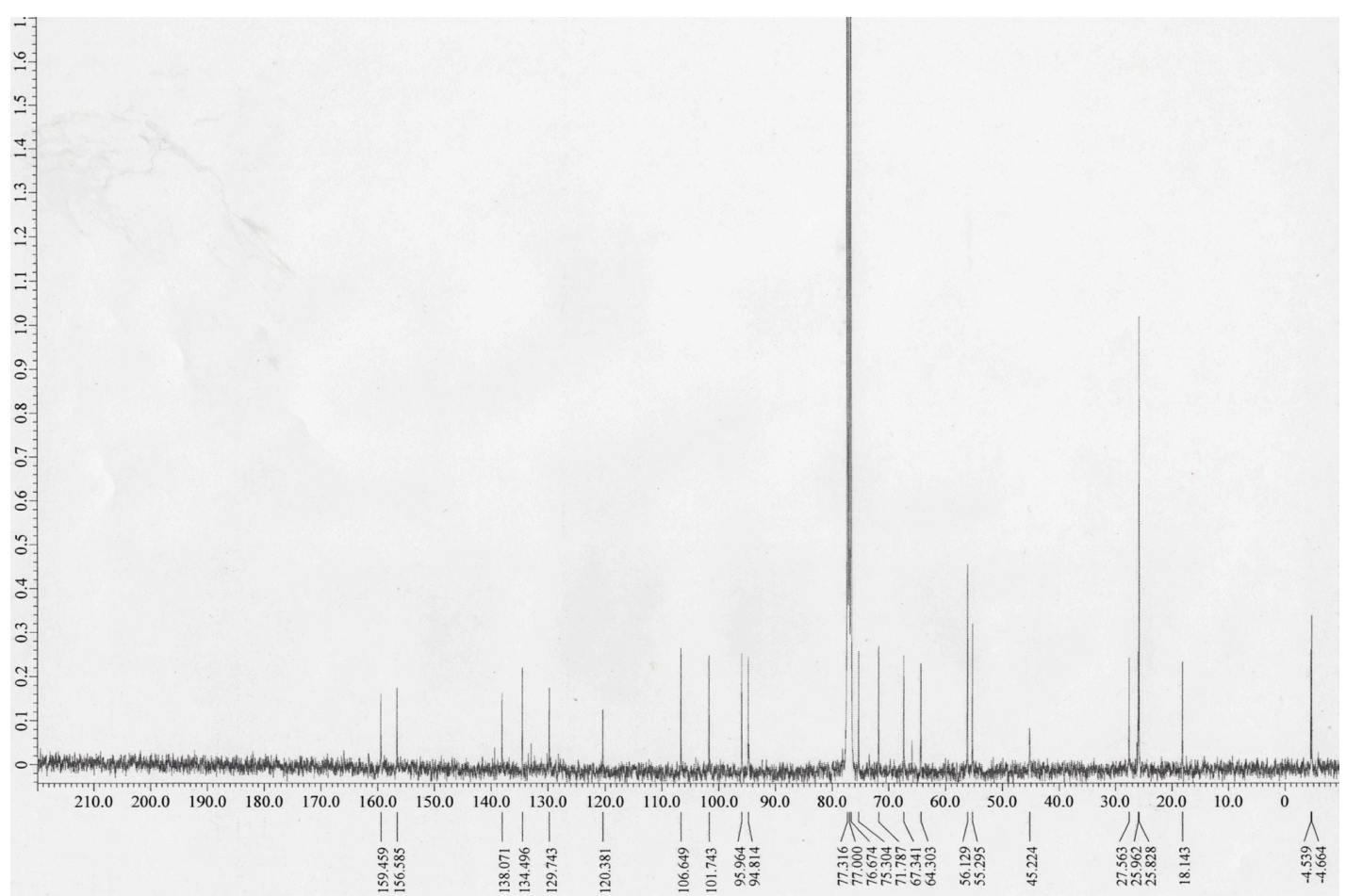




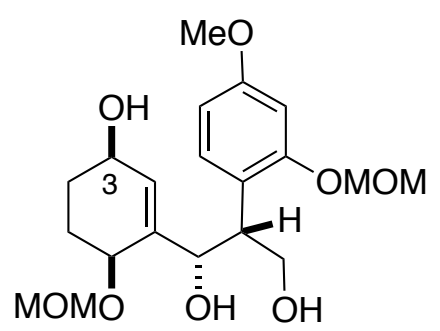

25

(5:1 mixture at $\mathrm{C} 3$ )

$\left(400 \mathrm{MHz}, \mathrm{CDCl}_{3}\right)$

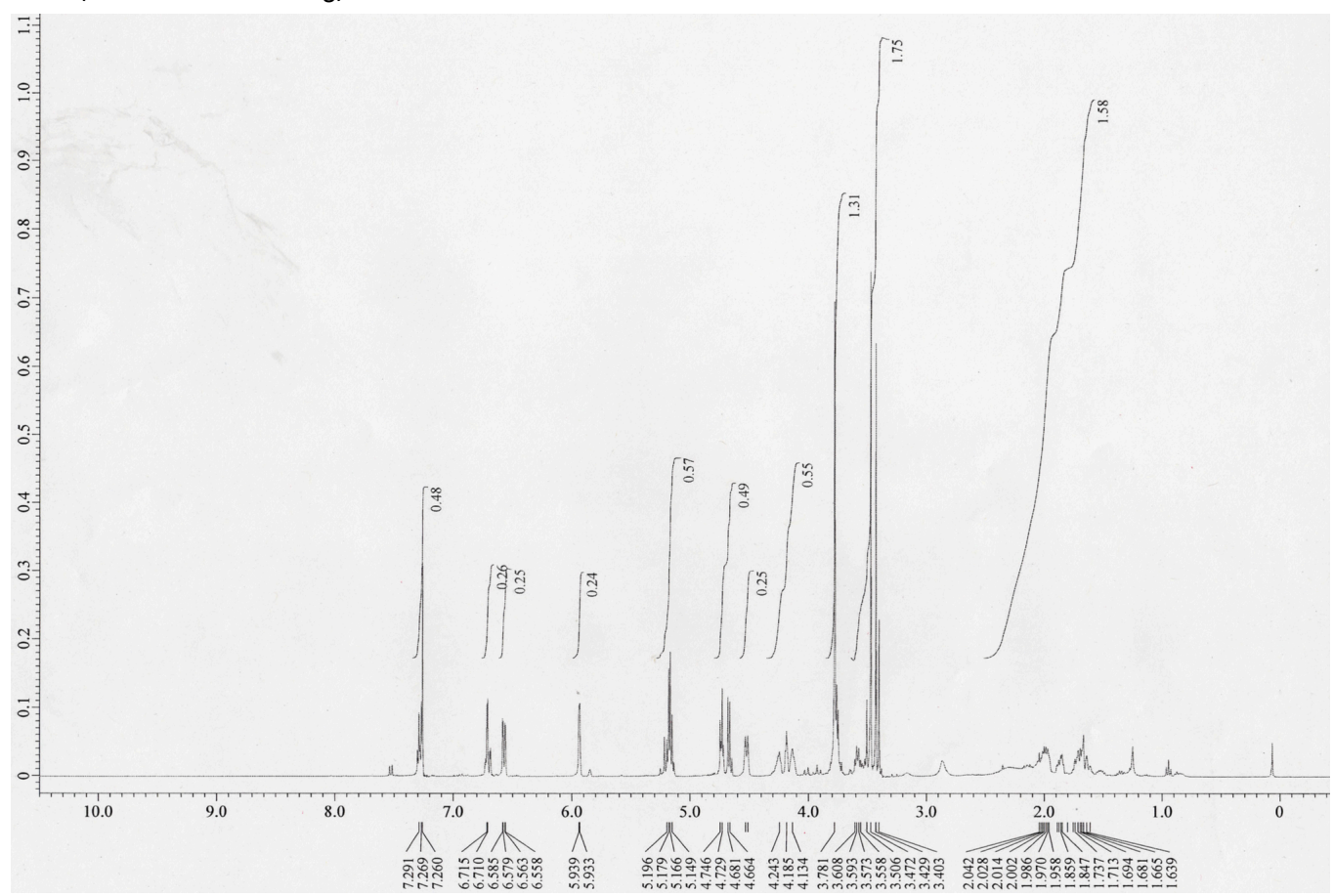




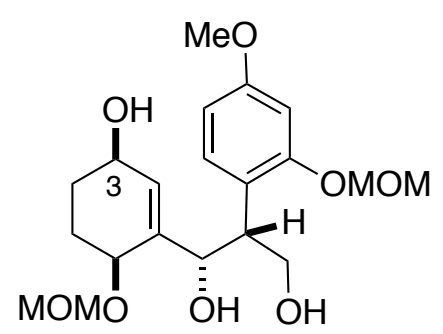

25

(5:1 mixture at $\mathrm{C} 3$ )

$\left(400 \mathrm{MHz}, \mathrm{CDCl}_{3}\right)$

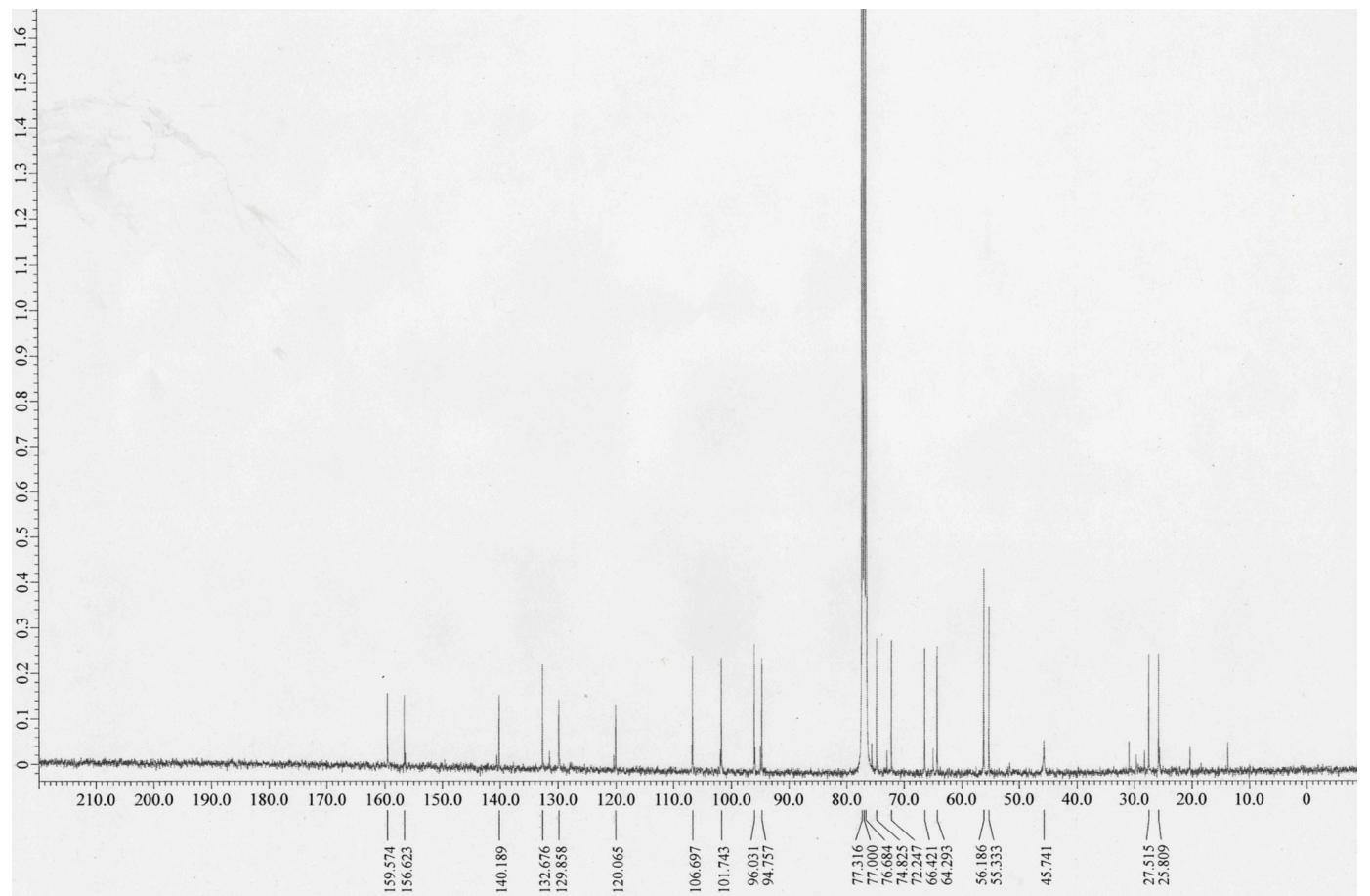



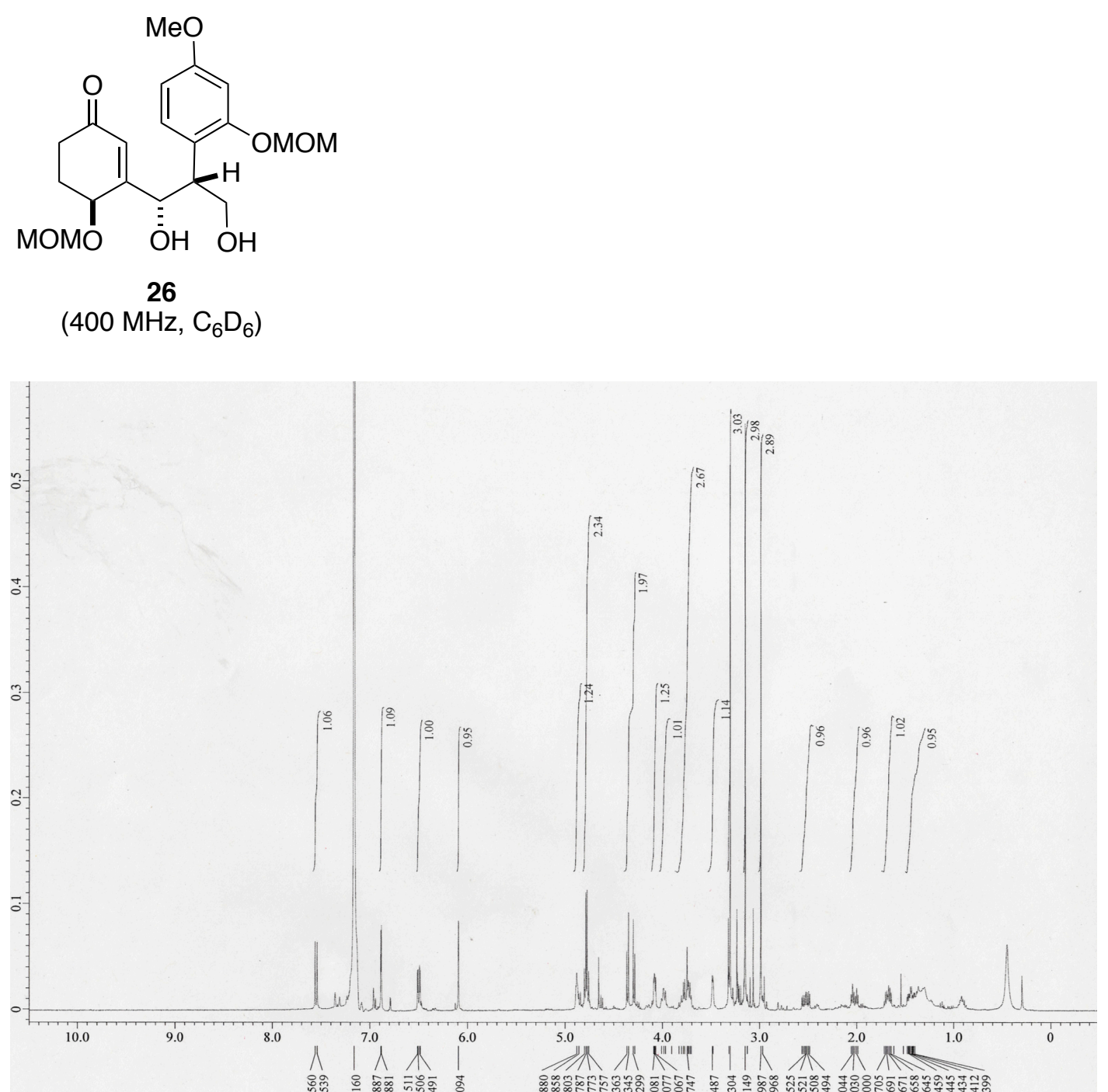

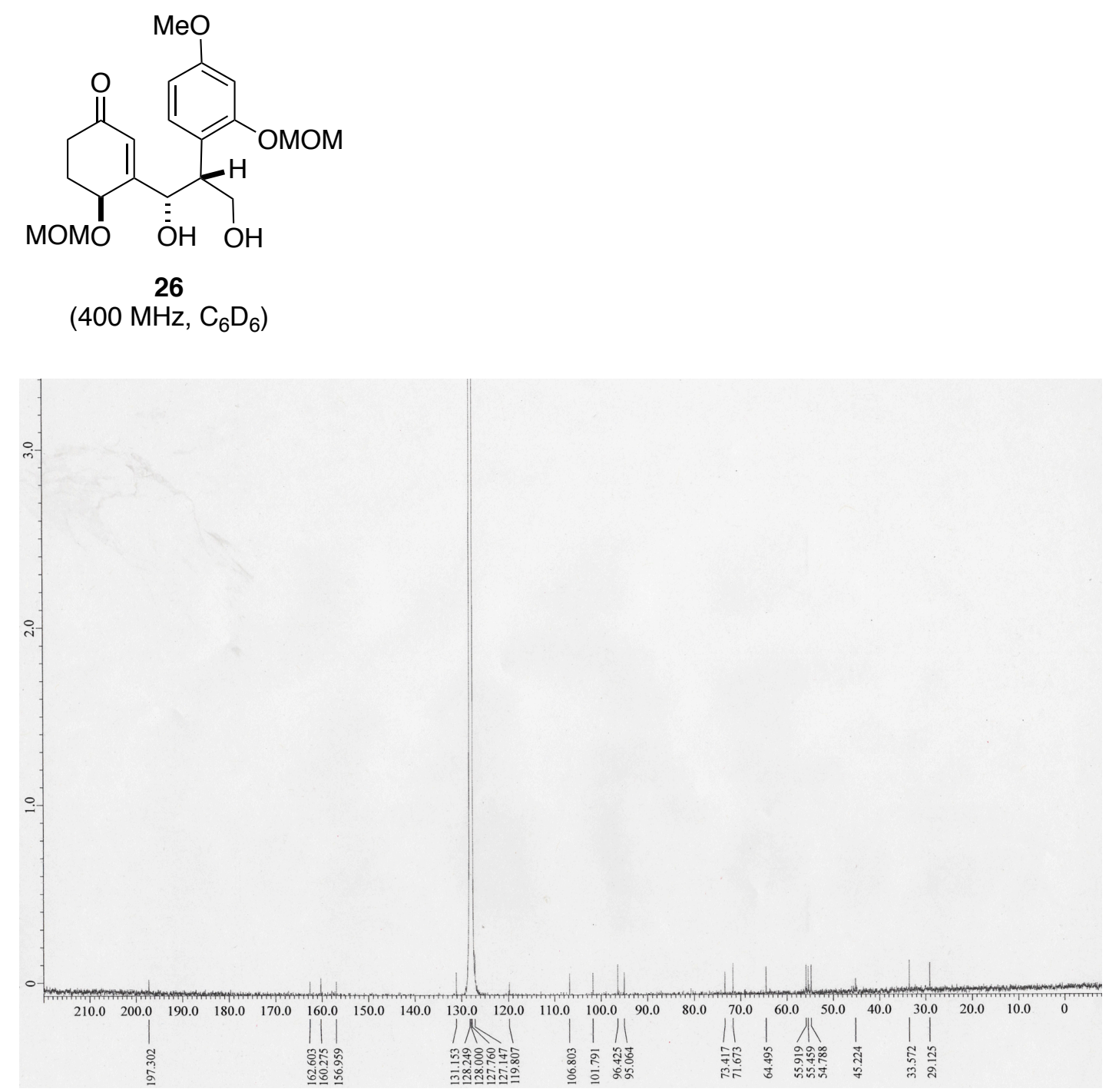


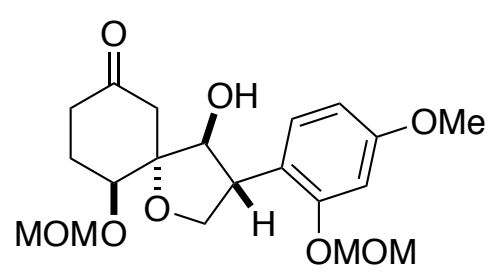

27

(single isomer after

column separation)

(400 MHz, $\mathrm{C}_{6} \mathrm{D}_{6}$ )

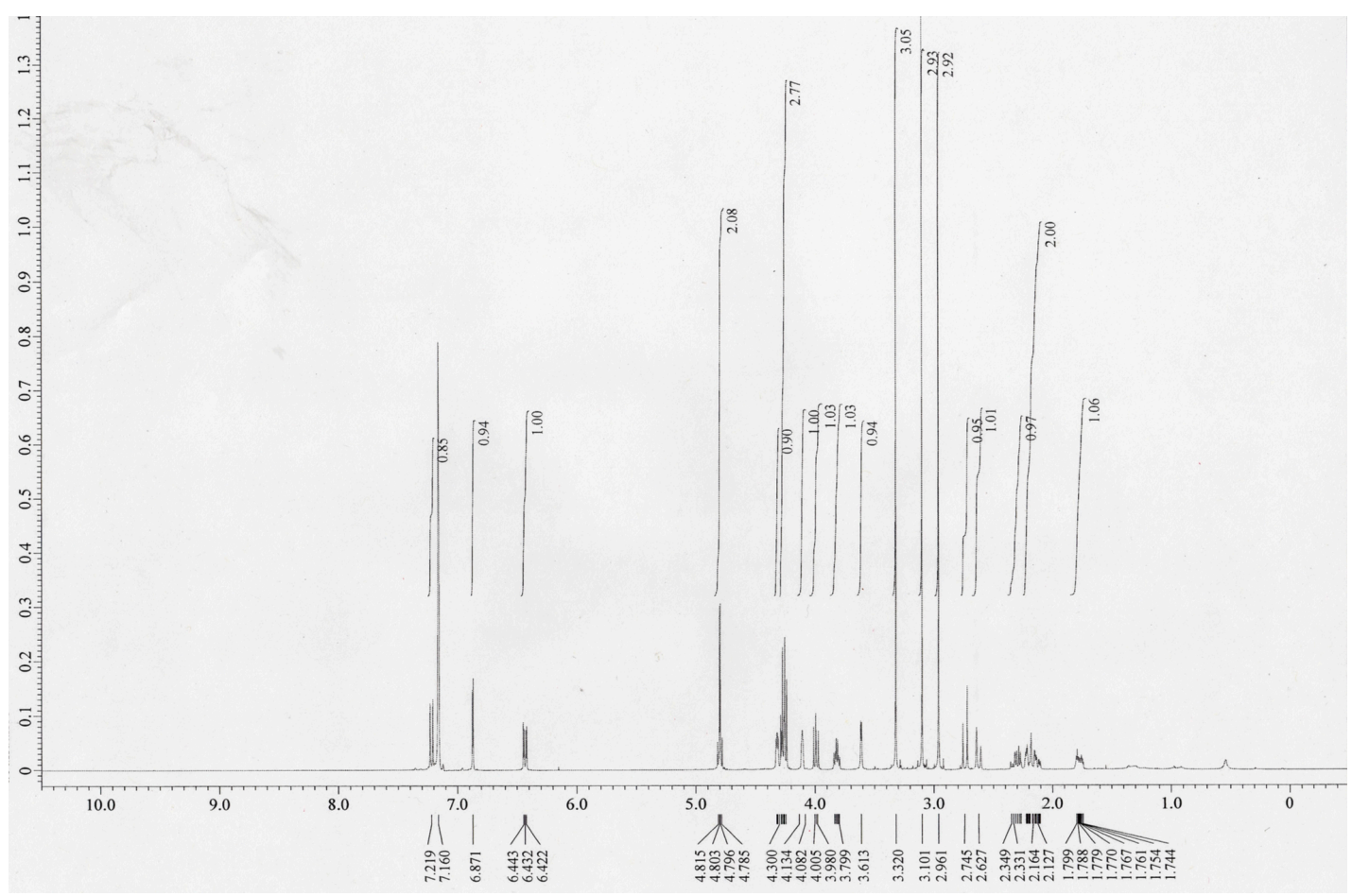




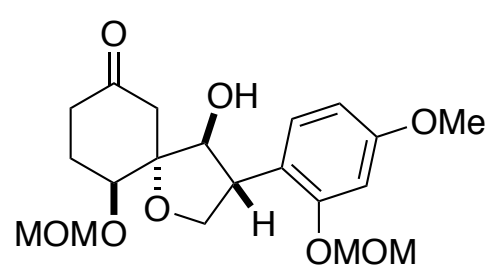

27

(single isomer after

column separation)

(400 MHz, $\mathrm{C}_{6} \mathrm{D}_{6}$ )

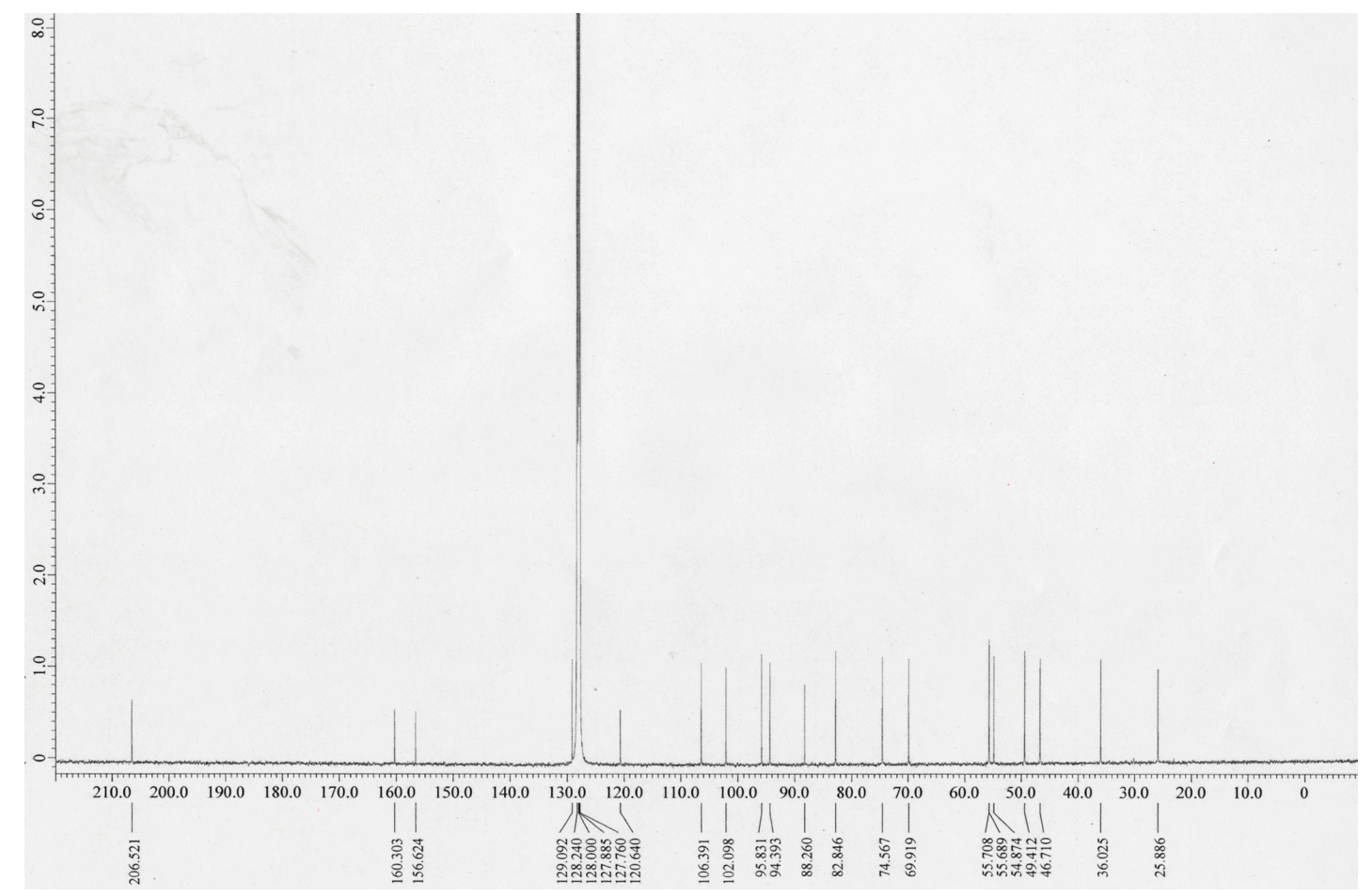


<smiles>COc1ccc([C@H]2CO[C@]3(CC(=O)CCC3OC)C2OC)c(OC)c1</smiles>

S3

(400 MHz, $\mathrm{C}_{6} \mathrm{D}_{6}$ )

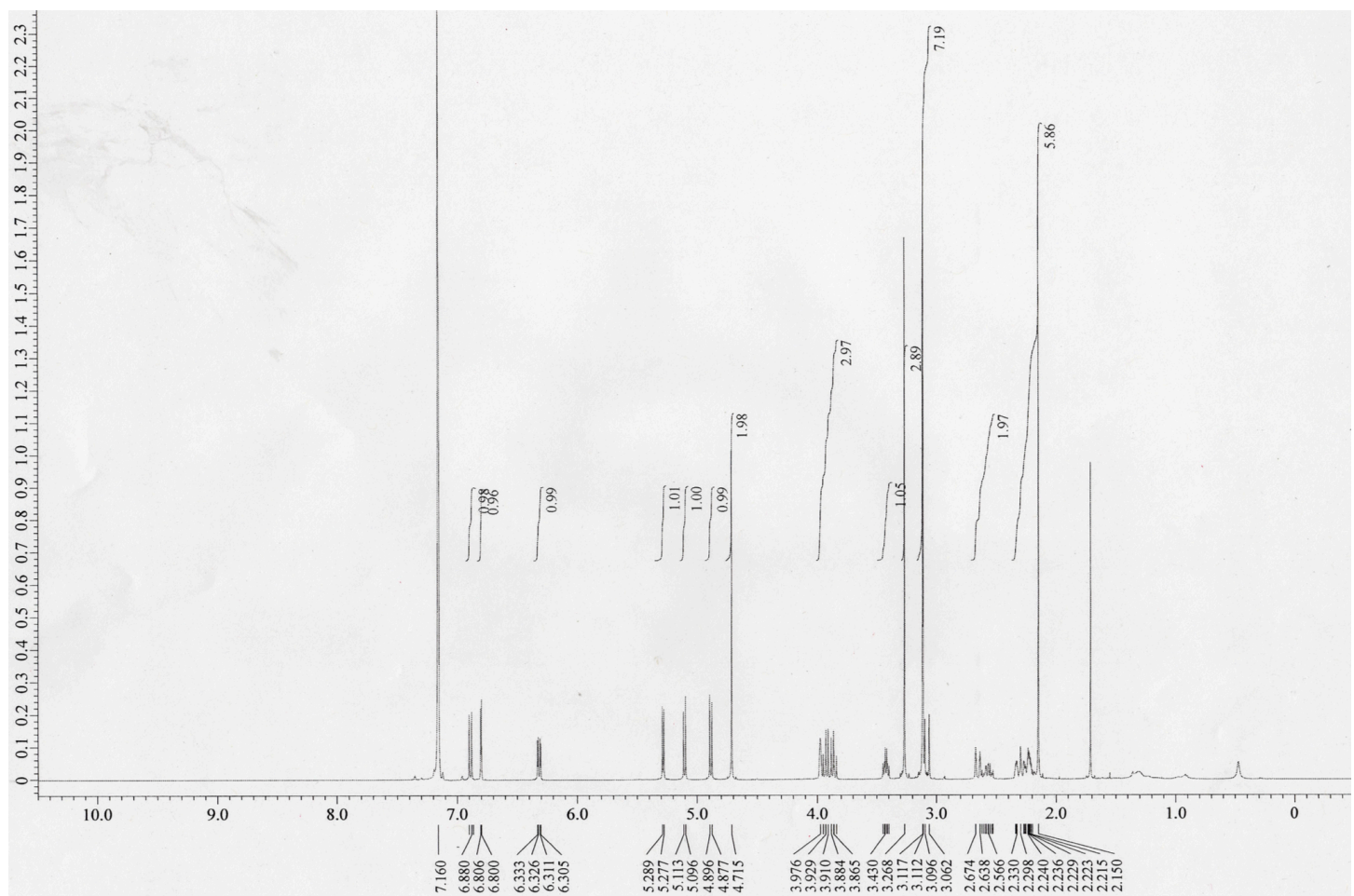


<smiles>COc1ccc([C@H]2CO[C@]3(CC(=O)CCC3OC)C2OC)c(OC)c1</smiles>

S3

(400 MHz, $\mathrm{C}_{6} \mathrm{D}_{6}$ )

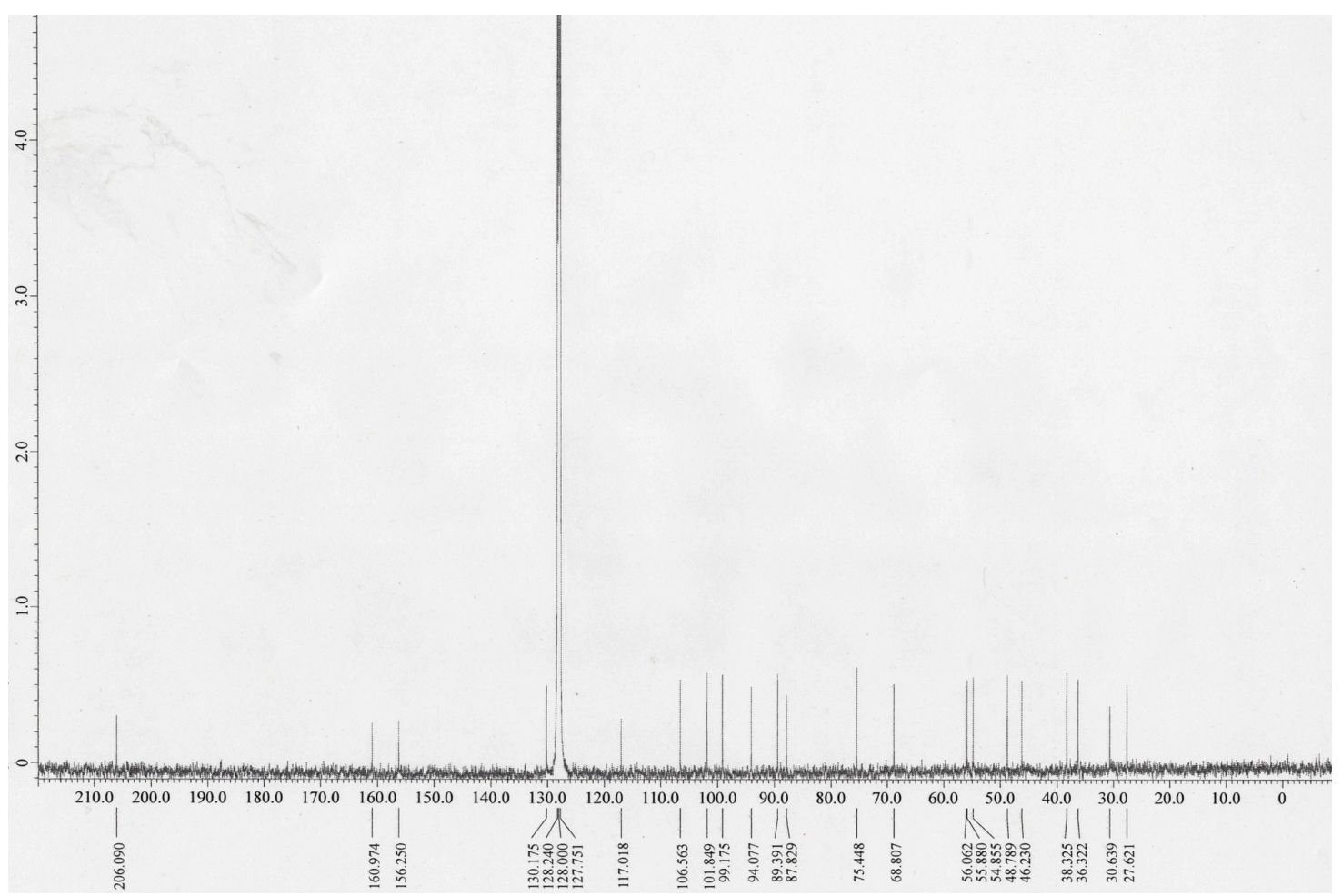




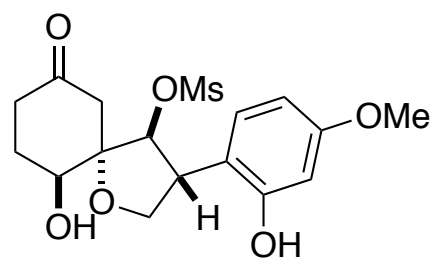

28

(400 MHz, $\left.\mathrm{CD}_{3} \mathrm{OD}\right)$

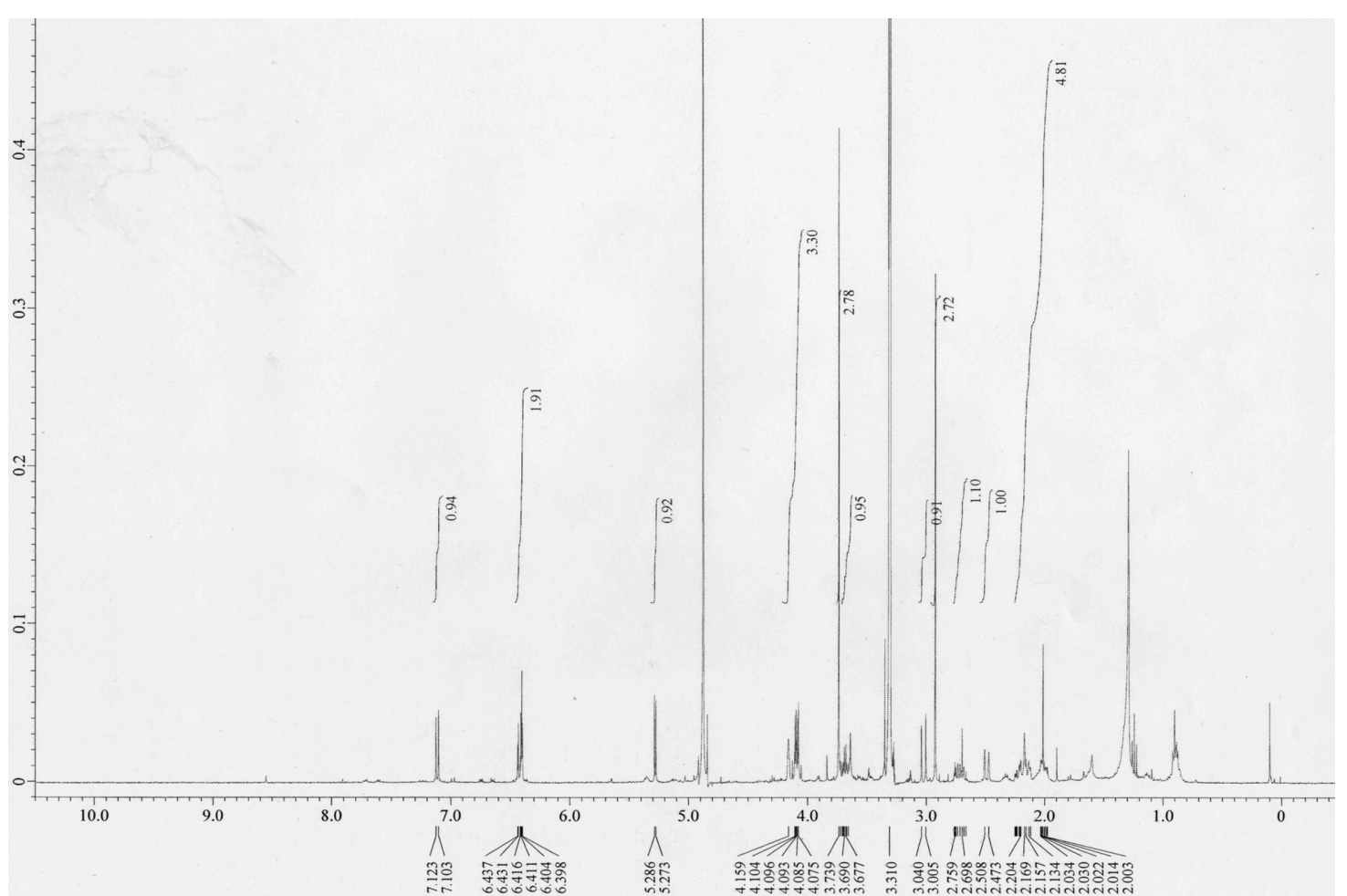




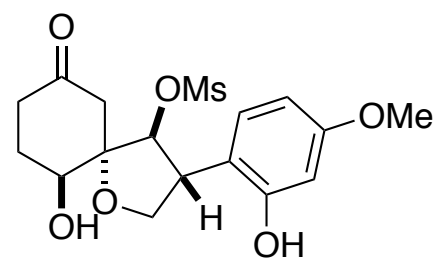

28

(400 MHz, $\mathrm{CD}_{3} \mathrm{OD}$ )

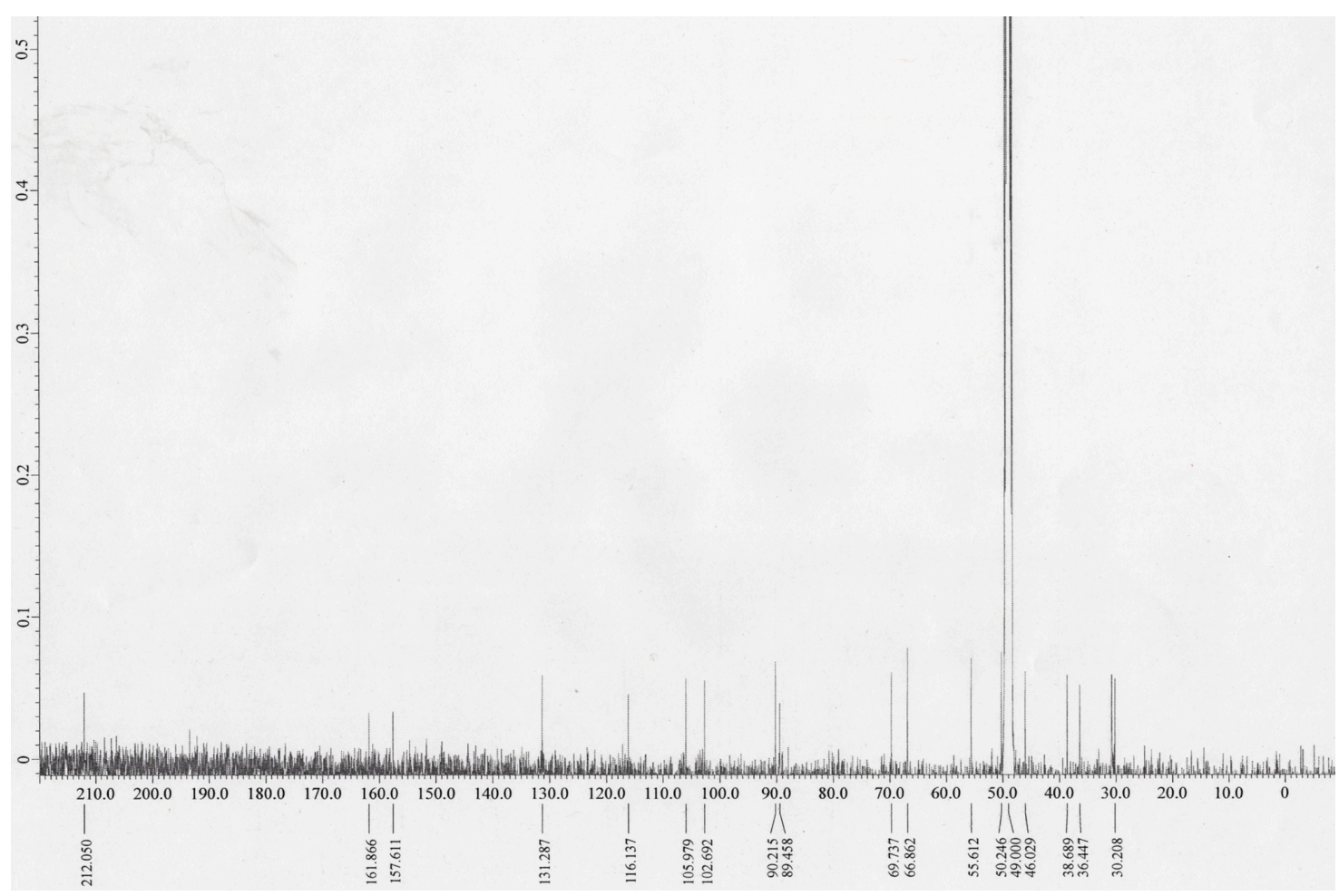


$\underbrace{O}_{\mathrm{HO}}$

stachyodin A (racemic 1)

(400 MHz, $\left.\mathrm{CD}_{3} \mathrm{OD}\right)$

upper: synthetic, bottom: literature for ${ }^{1} \mathrm{H}$ NMR

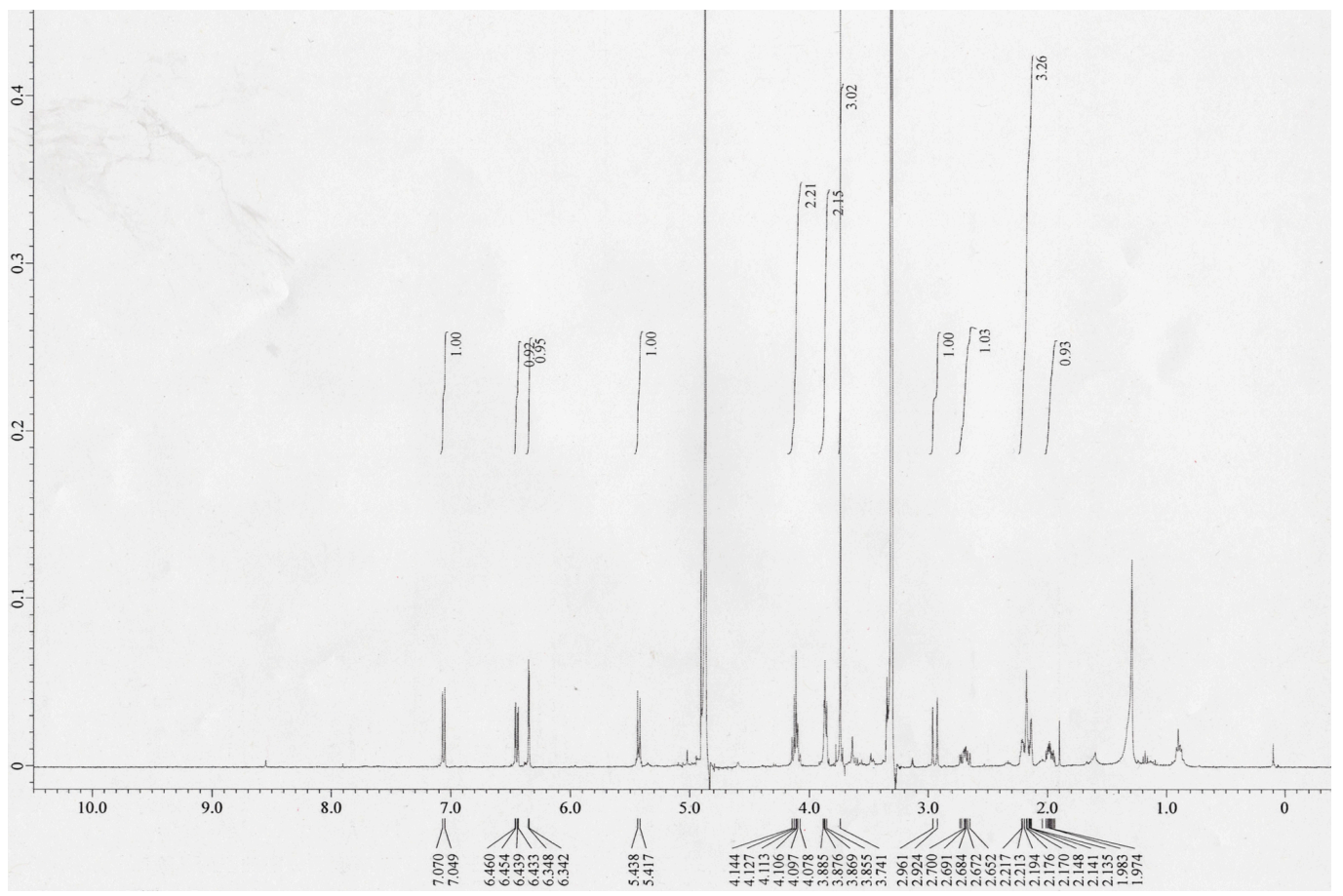

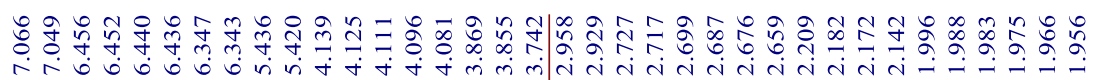

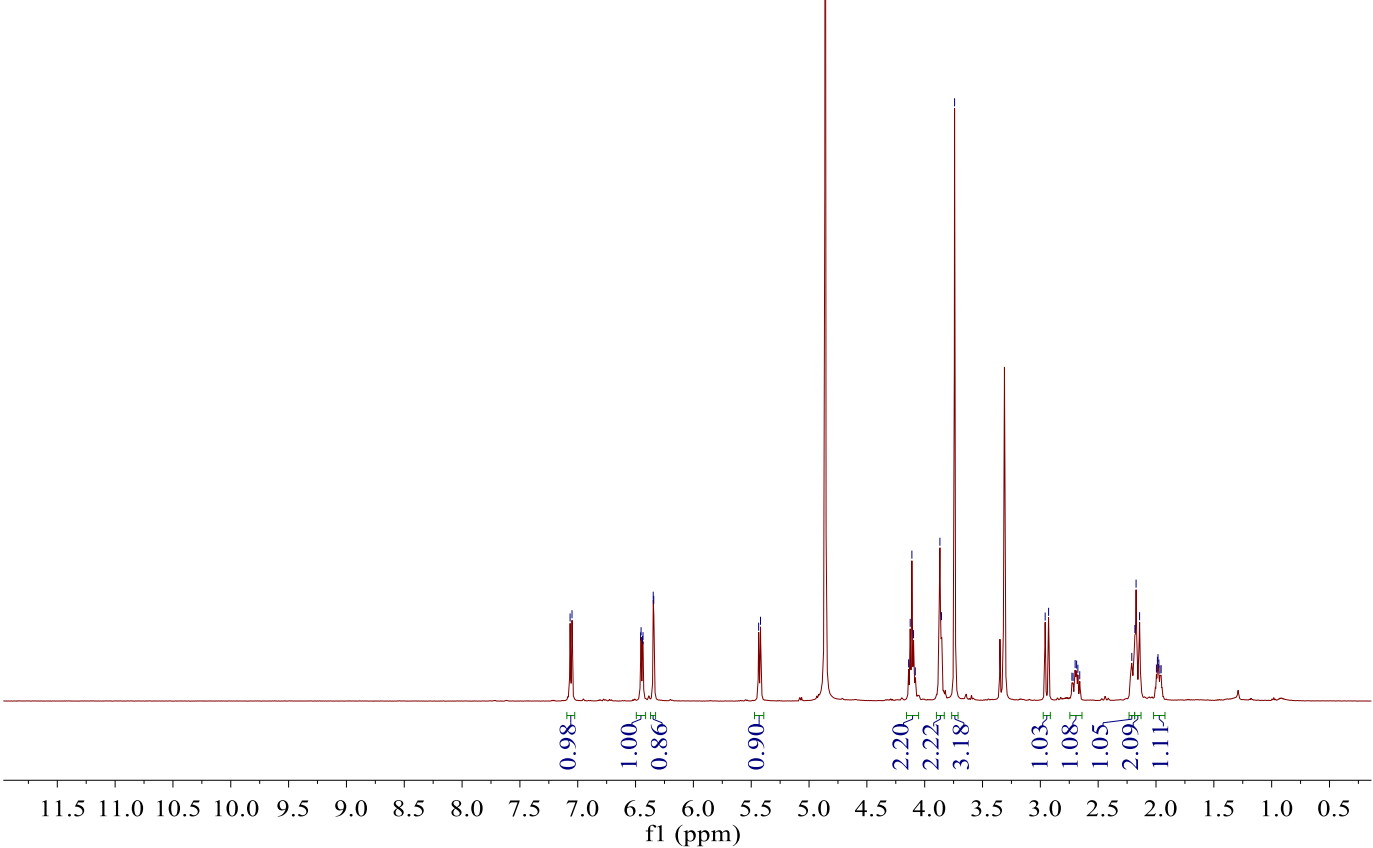


$\underbrace{O}_{\mathrm{HO}}$

stachyodin $\mathrm{A}($ racemic 1$)$
$\left(400 \mathrm{MHz}, \mathrm{CD}_{3} \mathrm{OD}\right)$

stachyodin $\mathrm{A}($ racemic
$\left(400 \mathrm{MHz}, \mathrm{CD}_{3} \mathrm{OD}\right)$

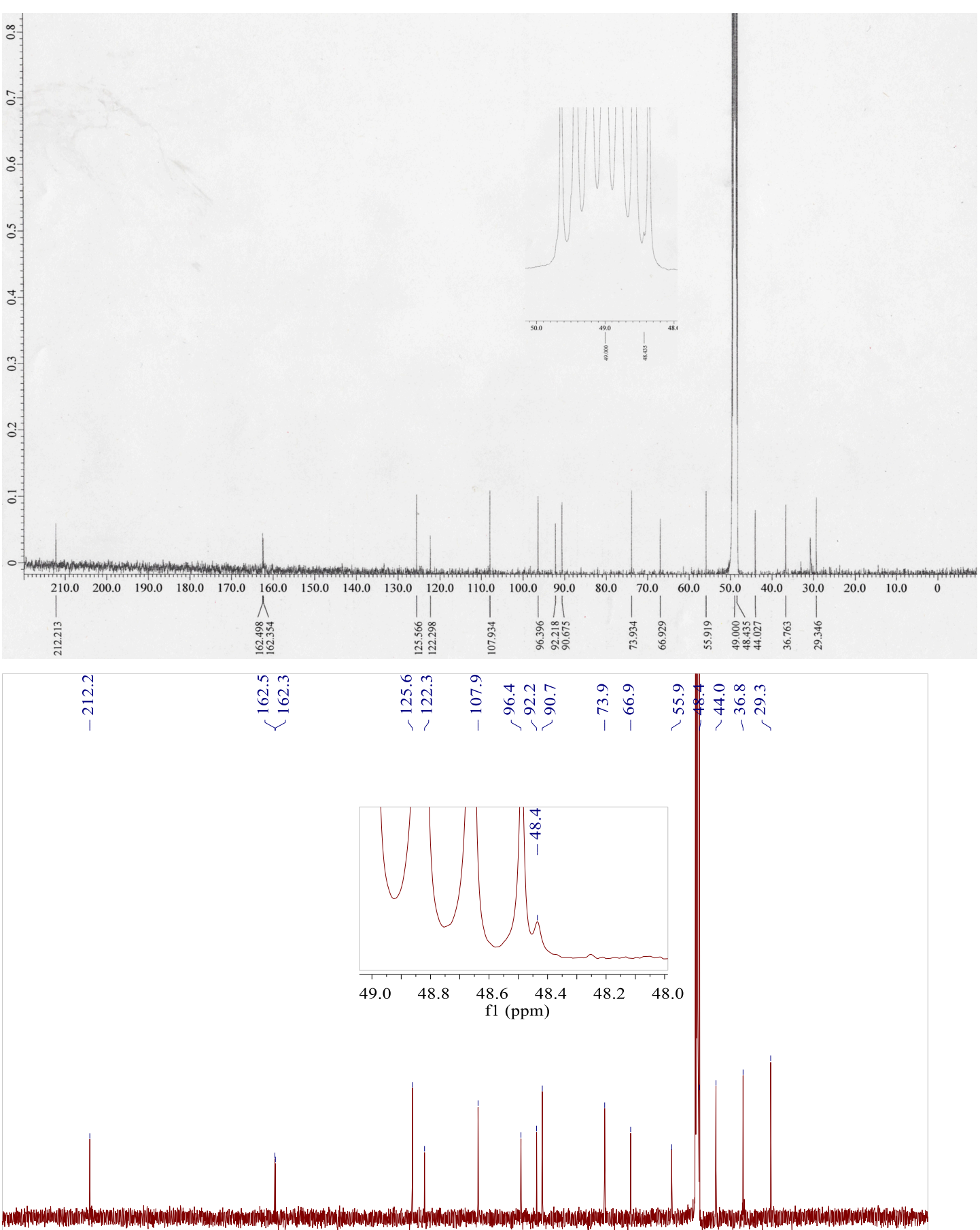

upper: synthetic, bottom: literature for ${ }^{13} \mathrm{C}$ NMR

$\begin{array}{lllllllllllllllllllllllll}230 & 220 & 210 & 200 & 190 & 180 & 170 & 160 & 150 & 140 & 130 & 120 & 110 & 100 & 90 & 80 & 70 & 60 & 50 & 40 & 30 & 20 & 10 & 0 & -1(\end{array}$ f1 (ppm) 
NOESY spectra of major isomer of compounds S2, 27 and S4
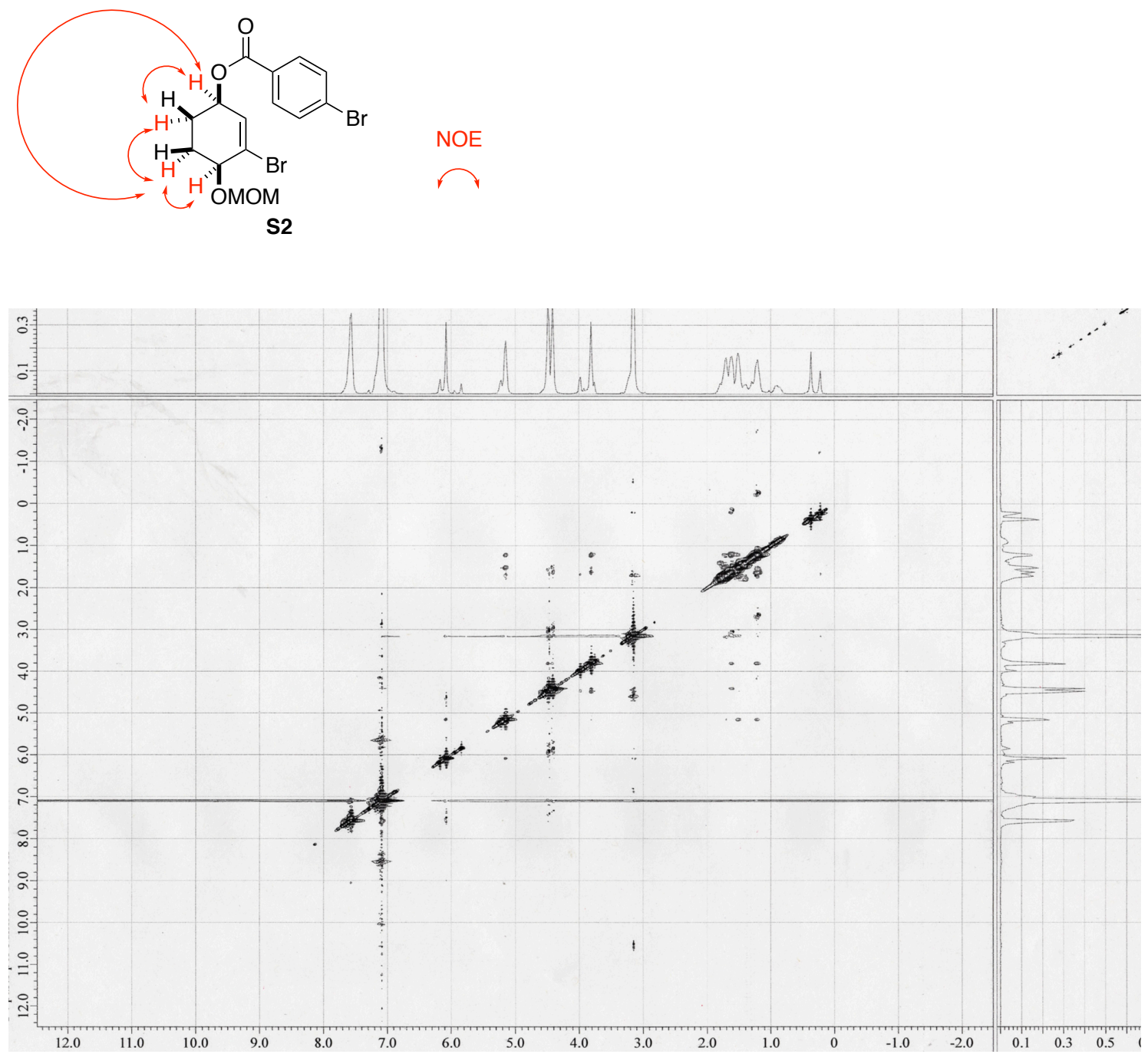


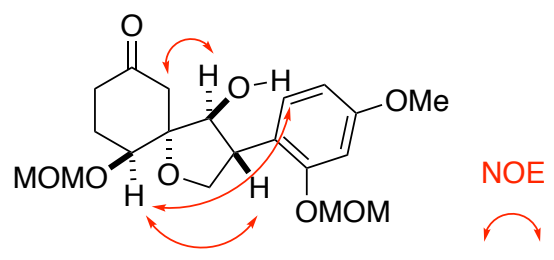

27

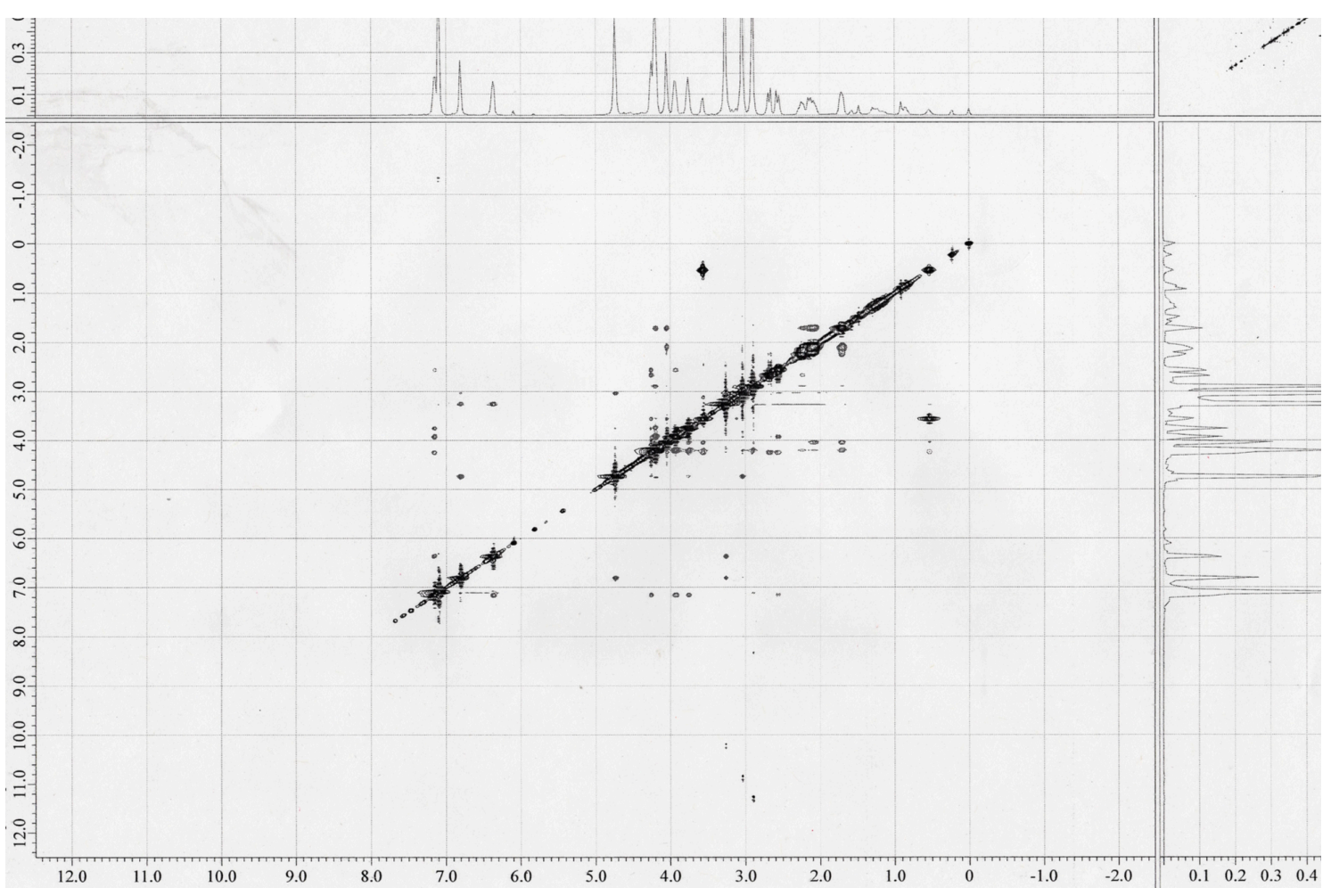




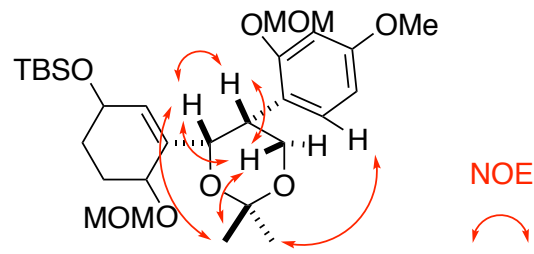

S4

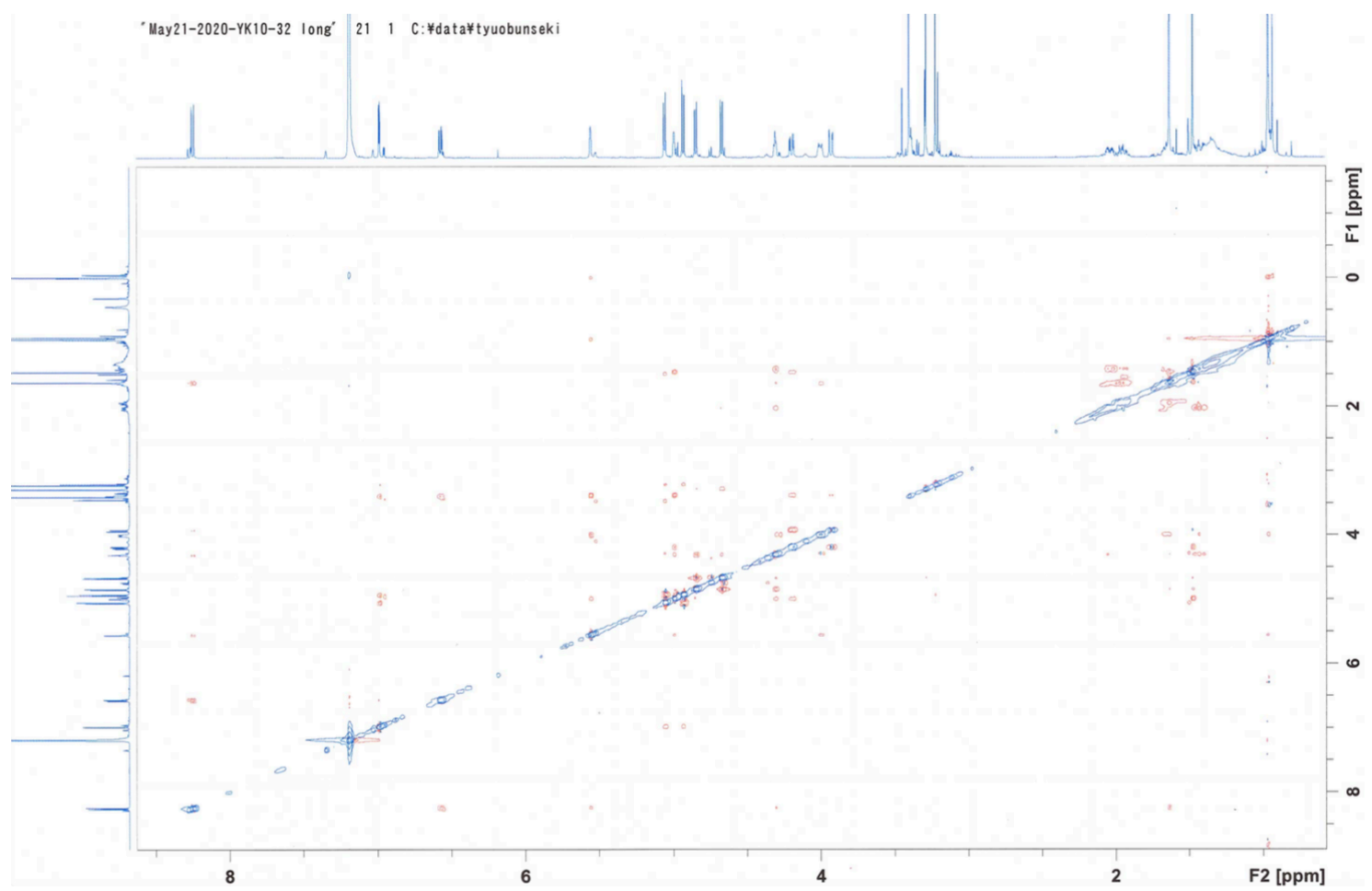

